\title{
Optical Observations of Disorder in Solid Helium 4
}

\author{
Satoshi Sasaki · Frédéric Caupin • \\ Sébastien Balibar
}

Published online: 15 October 2008

(C) Springer Science+Business Media, LLC 2008

\section{Erratum to: Journal of Low Temperature Physics, Volume 153, Nos. 3/4, November 2008 \\ DOI: $10.1007 /$ s10909-008-9824-4}

The paragraph breaks throughout the original article inadvertently disappeared during production. The publisher regrets the error.

The article is reprinted in its entirety on the following pages.

The online version of the original article can be found under doi:10.1007/s10909-008-9824-4.

S. Sasaki · F. Caupin · S. Balibar $(\bowtie)$

Laboratoire de Physique Statistique de l'Ecole Normale Supérieure, Associé au CNRS et aux Universités Paris 6 et Paris 7, 24 rue Lhomond, 75231 Paris, France

e-mail: balibar@lps.ens.fr 
Abstract It has been proposed that the supersolidity of helium 4 is associated with disorder in the solid samples. We report optical observations of this disorder in samples grown by different methods. We show that, when grown by the "blocked capillary method" as in most experiments showing anomalous phenomena, solid helium 4 can be polycrystalline with grain sizes down to the micrometer range, much smaller than the $0.1 \mathrm{~mm}$ found in some other experiments.

We analyze the properties of grain boundaries, and in particular the existence of liquid channels at the contact of grain boundaries with walls. Our analysis includes measurements of the contact angle of the liquid-solid interface with these walls, which exhibits a large hysteresis. Furthermore, we predict that similar channels should exist at the junction between grain boundaries.

Keywords Supersolids · Crystal growth · Disorder, Grain boundaries · Superfluid helium $4 \cdot$ Droplets, films, interfaces and wetting

PACS 67.80.-s $\cdot$ 61.72.Mm $\cdot$ 67.80.bf $\cdot 67.80 . \mathrm{bd} \cdot 68.08 . \mathrm{Bc} \cdot 67.25 . \mathrm{dg}$

\section{Introduction}

Since Kim and Chan's discovery [1-3] of anomalies in the low temperature properties of a torsional oscillator (TO) containing solid helium 4, the issue of supersolidity has become rather controversial [4]. Whatever the precise interpretation of these anomalies, it is generally accepted that they are associated with disorder in the solid samples. As a consequence, it is necessary to study this disorder in solid helium 4 as a function of the growth conditions. Several methods will have to be used for a complete study. Here we present optical observations which are adapted to defects larger than a micrometer, and having some density contrast with the average background solid. These defects are grain boundaries and liquid regions in the presence of the cell walls.

Let us first briefly summarize how disorder was shown to play an important role in the observed phenomena. At the beginning of their study, Kim and Chan grew all their samples at constant volume by the so-called "blocked capillary method" (BCM). Their results were reproduced by several groups [5-8], all using the same kind of TO and the same BCM. In 2006, Rittner and Reppy [5] showed that, in their experiments, annealing decreased the amplitude of the "non-classical rotational inertia fraction" (NCRIF) by more than one order of magnitude. Annealing is a well known method in materials science to improve crystal quality. Rittner and Reppy also reported the measurement of a very large NCRIF (up to 20\%) in rapidly quench-frozen samples where the amount of disorder was expected to be larger [9].

Grain boundaries (GBs) are common defects in crystals, and our group has studied their properties in helium. In 2006, Sasaki et al. [10, 11] showed that dc-mass superflow occurred through solid helium 4 at the liquid-solid coexistence only in the presence of GBs. In 2007, Sasaki et al. [12] observed that GBs are thin layers of microscopic thickness which are not completely wet by liquid helium 4 , even 
at the liquid-solid equilibrium. Sasaki's findings agree with the predictions by Pollet et al. [13, 14], who further predicted that GBs are superfluid except in special crystalline directions. However, Sasaki et al. [12] explained that no strong experimental evidence has been found yet for superfluidity along GBs. Ray and Hallock [15] observed flow in a cell containing solid helium off the melting curve but their present data do not prove that the flow occurred along GBs nor inside dislocation cores [16].

The recent study by Clark, West and Chan [17] showed that annealing samples grown by the BCM decreased the NCRIF down to a low but measurable level, comparable to the NCRIF of samples grown slowly at constant pressure and temperature from the superfluid. In the latter case, one expects solid samples to be single crystals [19]. However, for the growth of these single crystals, Clark et al. used two cells with similar shapes and different materials: they gave NCRIF differing by one order of magnitude $(0.04 \%$ in one cell, $0.4 \%$ in the other). As a consequence, it appears that, even in single crystals, the magnitude of the NCRIF may depend on uncontrolled disorder, for example a variable density of dislocations. ${ }^{1}$ Several models have already been proposed for the existence of supersolidity mediated by dislocations [21-23]. After discussions with M.H.W. Chan [18], we have realized that it might also depend on the crystal orientation.

Recently, Day and Beamish [24] observed a low temperature rise in the shear modulus of solid helium 4 samples grown by the BCM. They also found some effects of annealing. Moreover, their results are very sensitive to the helium 3 concentration down to the ppb level, as in TO measurements [25, 26]. This sensitivity was interpreted $[24,26]$ as evidence that, at low enough temperature, helium 3 atoms bind to the defects which are responsible for the observed anomalies.

As summarized in a recent review article [4], it is well established that disorder is involved in the various anomalies observed, but this disorder is not necessarily the same in samples prepared differently, and the exact mechanism by which it affects the properties of solid helium 4 is not yet clear. Given this situation, it has become important to study disorder in solid helium 4 samples grown by different methods.

In Sect. 2, we describe our experimental setup. In Sect. 3, we report observations on solid samples with different growth history, and show that the BCM leads to polycrystals, with grain sizes down to the micrometer range. In Sect. 4, we focus on the properties of GBs emerging at the liquid-solid interface. Measurements of contact angles with walls made of glass, copper, and graphite are reported in Sect. 5: a large hysteresis between advancing and receding interfaces is observed. In Sect. 6, we show that the contact line of a GB with a wall is generally a liquid channel. We present a detailed model explaining this observation, and we further predict the existence of similar channels at the junction between the GBs themselves. The consequences of the existence of such channels on mass-flow measurements are considered in Sect. 7.

\footnotetext{
${ }^{1}$ After growth at constant pressure and temperature, Clark's samples were cooled down at constant volume away from the liquid-solid equilibrium [17]. During this process, it is likely that non-hydrostatic stresses appear and create defects (dislocations, grain boundaries...). The density of these defects, consequently the NCRIF, should depend on the crystallization temperature. As far as we know, this possible dependence has not yet been studied.
} 


\section{Experimental Setup}

Figure 1 shows a typical helium cell which can be seen through the windows of our optical cryostat. The cell has a square cross section and it is made by closing a $11 \times 11 \mathrm{~mm}$ hole in a thick copper plate (B) with two glass windows (A). The windows are $6 \mathrm{~mm}$ thick and $25 \mathrm{~mm}$ in diameter. The cell is leak-tight thanks to indium rings between the windows and the copper body. A $10 \mathrm{~mm}$ thick cell was used to make general observations of crystallization either from normal liquid helium 4 or from superfluid helium 4. Another cell ( $3 \mathrm{~mm}$ thick) enabled us to observe single GBs and the groove they make when emerging at the liquid-solid interface. In such a thinner cell, the grains are usually side by side and their boundaries easier to observe because roughly parallel to the optical axis, that is perpendicular to the windows.

\subsection{Samples Grown by the "Blocked Capillary Method" (BCM)}

A capillary $(0.6 \mathrm{~mm}$ inner diameter and $50 \mathrm{~mm}$ long) connects the thick cell to a capacitance pressure gauge (C). However, it should be noted that this gauge did not allow us to monitor the exact pressure in the cell when solid was present, because mass could not flow freely along the capillary [27]. The cell temperature is measured with a carbon-resistance thermometer (D) and a germanium thermometer (E) on (A) and regulated thanks to a heater $(\mathrm{F})$. The cell is mounted on a gold plated copper piece (G) attached to the mixing chamber of the dilution refrigerator above. Inside the thin cell, a small glass piece was glued at the top in order to study contact angles of the liquid-solid interface with glass, not only with copper. Samples were illuminated with white parallel light through windows and images were taken from the side opposite to the optical source with a CCD camera outside the cryostat. The fill line $(\mathrm{H})$ has a

Fig. 1 (Color online) Overview of an optical cell in a copper plate closed with two glass windows. A, glass window; B, copper plate with square hole; C, Straty-Adams type capacitance pressure gauge; D, carbon-resistance thermometer;

E, germanium-resistance thermometer; $\mathbf{F}$, heater; $\mathbf{G}$, copper piece; $\mathbf{H}$, fill line

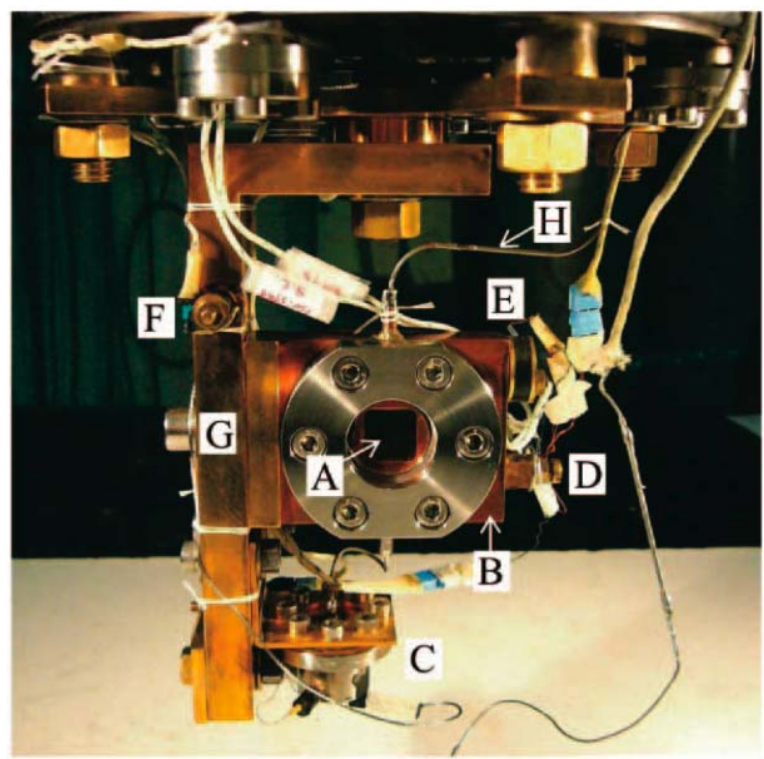


$0.6 \mathrm{~mm}$ inner diameter close to the cell. It connects the cell to a cylinder outside containing natural purity helium 4 . It is commonly accepted that natural purity helium 4 contains helium 3 impurities with a concentration of about $0.3 \mathrm{ppm}$.

\section{Sample Morphology}

In this section, we present general observations of crystals grown either by the BCM from the normal liquid, or by fast injection of mass at low temperature where the liquid is superfluid.

The BCM works as follows when a dilution refrigerator is used, which is the case in most of the recent experiments addressing supersolidity. The cell is filled with normal liquid helium 4 at high pressure, typically $5 \mathrm{MPa}$ or more, at a temperature which is higher than the crystallization temperature everywhere along the fill line (typically above $3 \mathrm{~K}$ ). In order to cool down, one usually starts by pumping the " $1 \mathrm{~K}$ pot". Since the fill line is thermally anchored to the pot, a solid plug forms there, which isolates a certain mass of helium inside the cell from the outside. Assuming that this plug does not move and is really leak-tight, solidification takes place at constant volume in the cell when cooling proceeds during the circulation of the mixture in the dilution unit. The liquid in the cell follows an isochore which hits the melting line at a temperature $T_{\mathrm{i}}$. It is often assumed that $T_{\mathrm{i}}=T_{\mathrm{m}}\left(P_{\mathrm{i}}\right)$, where $P_{\mathrm{i}}$ is the initial pressure at which the cell was filled. In principle, the pressure in the cell leaves the melting curve when it is full of solid, at a temperature $T_{\mathrm{f}}$ which is such that the molar volume of the crystal $V_{\mathrm{S}}\left(T_{\mathrm{f}}\right)$ equals the initial molar volume of the liquid $V_{\mathrm{L}}\left(T_{\mathrm{i}}\right)$. However, there are several approximations and difficulties in the above description because:

1. During the crystallization inside the part of the fill line between the cell and the $1 \mathrm{~K}$ pot, the pressure in the cell decreases, especially if the cell volume is not very large compared to that of this fill line section. As a consequence, the pressure in the cell does not exactly follow an isochore.

2. Even a true isochore is not constant in pressure. $P$ and $T$ are linked by the relation

$$
\left(\frac{\partial P}{\partial T}\right)_{V}=\frac{\alpha_{P}}{\chi_{T}}
$$

where $\alpha_{P}$ is the isobaric expansion coefficient and $\chi_{T}$ the isothermal compressibility. The slope of the liquid isochore in the $P-T$ plane thus varies approximately from 0.1 to $0.3 \mathrm{MPa} \mathrm{K}^{-1}$ between 2.5 and $13 \mathrm{MPa}$ [32].

3. The plug near the $1 \mathrm{~K}$ pot might move and it is not necessarily leak tight.

4. The crystal is not necessarily homogeneous in density since it is formed at variable pressure during the whole process.

5. Off the melting curve, liquid regions might persist because curvature effects or local stresses stabilize them (see Sect. 6.1).

6. If $P_{\mathrm{i}} \leq 4.9 \mathrm{MPa}$, not all the helium in the cell can be crystallized (see Sect. 3.3).

In practice, we have observed that crystallization starts in the cell at a temperature which is close to the estimate $T_{\mathrm{m}}\left(P_{\mathrm{i}}\right)$. It ends at a temperature $T_{\mathrm{f}}$ which is slightly higher than expected as we shall see for crystallization along three different paths which we have labeled A, B and C on the phase diagram (see Fig. 2). 
Fig. 2 Phase diagram in the volume-temperature plane (upper panel) and in the pressure-temperature plane (lower panel). Most of the data have been taken from Hoffer [28]. The melting curve at high temperature is from Swenson [29, 30] and the lambda line from Vignos and Fairbank [31]. For crystallization with the BCM, we have studied 3 different paths (A, B and $\mathbf{C})$
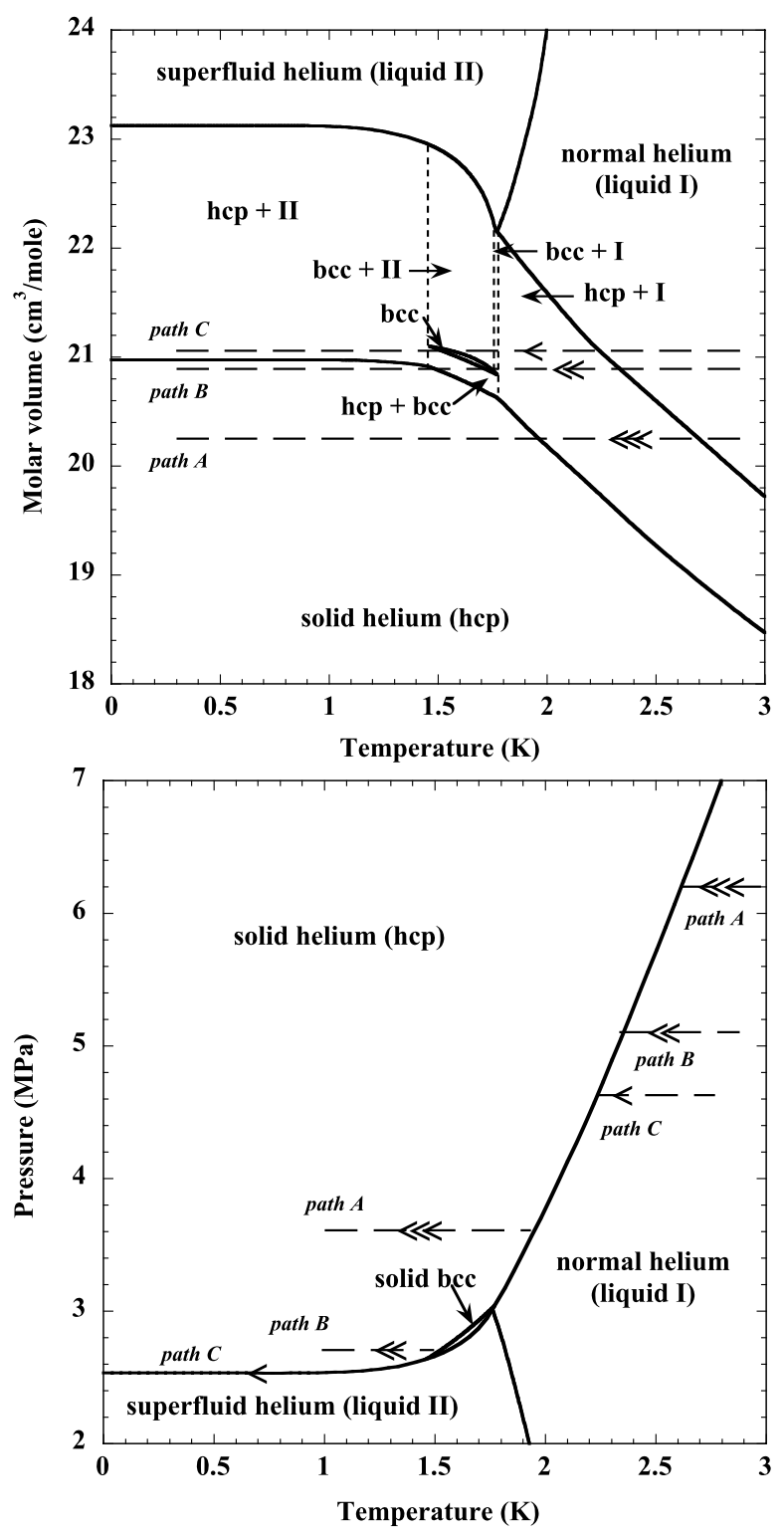

\subsection{Crystallization at High Pressure: Path A}

Figure 3 shows crystallization along path $\mathrm{A}$ in the thin cell. We have glued a glass piece on its top wall for observations of contact angles with glass (see Sect. 5). Two crosses carved on the windows (lower right corner) help adjusting the focusing of the CCD camera. We started by fixing the outside pressure at $P_{\mathrm{i}}=6.2 \mathrm{MPa}$ and crystallization started in the cell at $2.58 \mathrm{~K}$ with the appearance of hcp solid helium 4 on the copper walls of the cell, which are its coldest parts (Fig. 3a). According to 


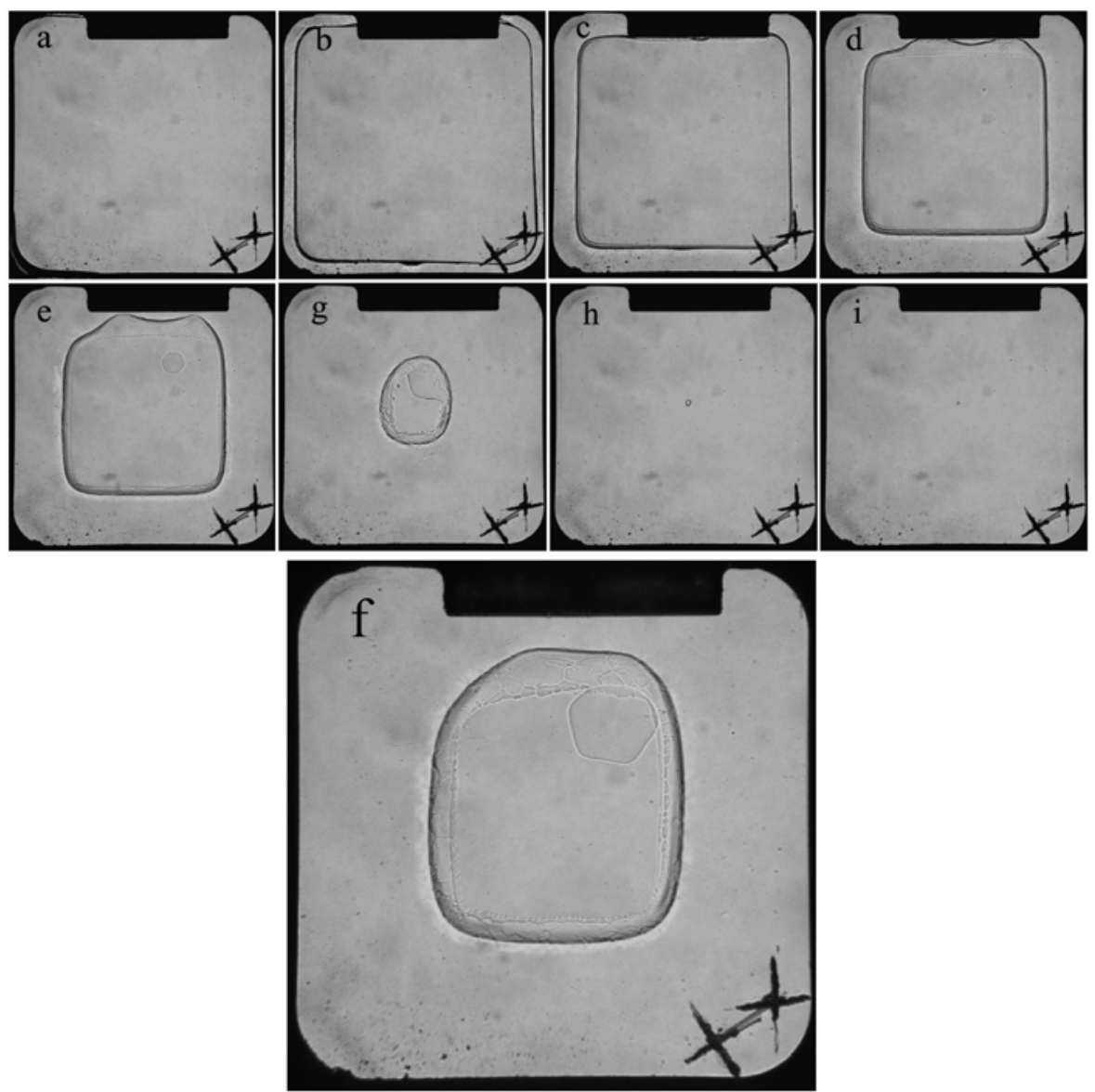

Fig. 3 Crystallization of hcp solid in the thin cell using the BCM along path A in Fig. 2. Crystallization started from the top and bottom corners on the left from normal liquid at $2.58 \mathrm{~K}$ (a) and solid slowly grew along the melting curve of helium 4 during cooling down $(\mathbf{b}-\mathbf{h})$. The cell was full of solid at $1.95 \mathrm{~K}$ after 3 hours (see i). The temperature interval between each image is roughly $100 \mathrm{mK}$. The cell is $11 \mathrm{~mm}$ wide by $11 \mathrm{~mm}$ high. A glass piece is attached at the top of the cell, and two crosses carved on the windows (lower right corner) help adjusting the focusing

a melting curve obtained by interpolating data from Hoffer [28] up to $2.2 \mathrm{~K}$ and from Swenson [29, 30] at 2.5, 3, 3.5 and $4 \mathrm{~K}$ (see Fig. 2), the melting temperature $T_{\mathrm{m}}(6.2 \mathrm{MPa})$ is $2.59 \mathrm{~K}$, close to our value.

After the first appearance of solid helium in the cell, crystallization proceeded towards the center (see Figs. $3 \mathrm{~b}$ to $3 \mathrm{~h}$, which are taken at temperature intervals of about $100 \mathrm{mK}$ ). Being cooled down from its sides, the cell is slightly warmer at the center, especially at relatively high temperature where neither the normal liquid nor the solid are good thermal conductors. In this particular run, the cell was full of hcp solid at $T_{\mathrm{f}}=1.95 \mathrm{~K}, 3$ hours after the beginning of the crystallization (Fig. 3i). This means that, inside the cell, crystallization was completed at $P_{\mathrm{m}}\left(T_{\mathrm{f}}\right)=3.6 \mathrm{MPa}$, that is $2.6 \mathrm{MPa}$ below the initial pressure. At $2.58 \mathrm{~K}$ on the 
melting curve, the molar volume of the liquid is $V_{\mathrm{L}}=20.44 \mathrm{~cm}^{3} \mathrm{~mol}^{-1}$. Consequently, one would expect the crystallization to complete at $1.86 \mathrm{~K}$ where $V_{\mathrm{S}}=$ $20.44 \mathrm{~cm}^{3} \mathrm{~mol}^{-1}$, instead of the observed $1.95 \mathrm{~K}$, where $V_{\mathrm{S}}=20.27 \mathrm{~cm}^{3} \mathrm{~mol}^{-1}$ (see Fig. 2). The difference is somewhat surprising. If no relaxation took place in the solid, the molar volume of the solid near the walls would be smaller than $20.44 \mathrm{~cm}^{3} \mathrm{~mol}^{-1}$, and larger at the center. In order to insure mass conservation, crystallization would then be complete a temperature lower than $1.86 \mathrm{~K}$, not higher. One might invoke a temperature difference between the cell walls and its center. Such a temperature difference is likely, as suggests the fact that, during crystallization (b to e), the liquid-solid interface follows an isotherm with a square shape similar to the cell itself. However, since the thermometer measures the temperature on the copper walls outside, one should have again observed the end of the crystallization at a temperature lower than $1.86 \mathrm{~K}$. It is possible that during crystallization, since the outside pressure is kept at $6.2 \mathrm{MPa}$ while the cell pressure is much lower, some mass enters the cell. Either the plug moves or it is slightly porous. Day and Beamish [33] observed mass flow in solid helium through an array of 36000 capillaries ( $25 \mu \mathrm{m}$ in diameter) at $1.95 \mathrm{~K}$ near melting, a phenomenon which they attributed to the existence of thermally activated vacancies whose activation energy is estimated to be $13 \mathrm{~K}$ [20]. It is thus possible that the plug allows some mass flow. It is also possible that some liquid regions persist in the cell or in the fill line, stabilized by curvature effects or by local stresses (see Sect. 6.1).

In the cell, the solid is transparent but it does not mean that it has no disorder. The shape of the liquid-solid interface is not distorted by defects at the beginning of the crystallization (Figs. $3 \mathrm{~b}$ to $3 \mathrm{~d}$ ), probably because the temperature gradient is large at that moment, and forces the interface to follow a smooth isotherm accurately. Figures $3 \mathrm{e}$ to $3 \mathrm{~g}$ show that some solid grows on the windows which must be colder than the inside of the cell. On these three figures, the liquid-solid interface shows a definite roughness which indicates that the solid is highly polycrystalline, something which we have confirmed by depressurizing the sample at low temperature (see Sect. 3.4 and Fig. 6).

Figures $3 \mathrm{e}$ and $3 \mathrm{f}$ also show evidence for the hexagonal symmetry of hcp crystallites which sometimes nucleate on the windows. The hexagonal crystal on figure $3 \mathrm{f}$ looks facetted but we doubt that its sides are really flat because the roughening transition $T_{R 2}$ for the side facets of hexagonal prisms is, in principle, around $1 \mathrm{~K}$ [19]. For these crystals to be really facetted at $2 \mathrm{~K}$, the roughening transition would need to be reentrant along the melting curve. This is perhaps possible if the solid-liquid interfacial tension $\sigma_{\mathrm{LS}}$ increases strongly with pressure along the melting line $P_{\mathrm{m}}(T)$ [34], but it would need to be carefully studied (see also Sect. 3.5). We note that an inverse roughening transition has recently been observed in carbon dioxide [35]. Another possibility is that $\sigma_{\mathrm{LS}}$ increases across the transition at $1.76 \mathrm{~K}$ where the superfluidity disappears.

\subsection{Crystallization across the hcp-bcc Transition: Path B}

Figure 4 shows crystallization along path $\mathrm{B}$. The initial pressure being $5.1 \mathrm{MPa}$, crystallization starts at $2.36 \mathrm{~K}$ (Fig. 4a). This is close again to the temperature expected 

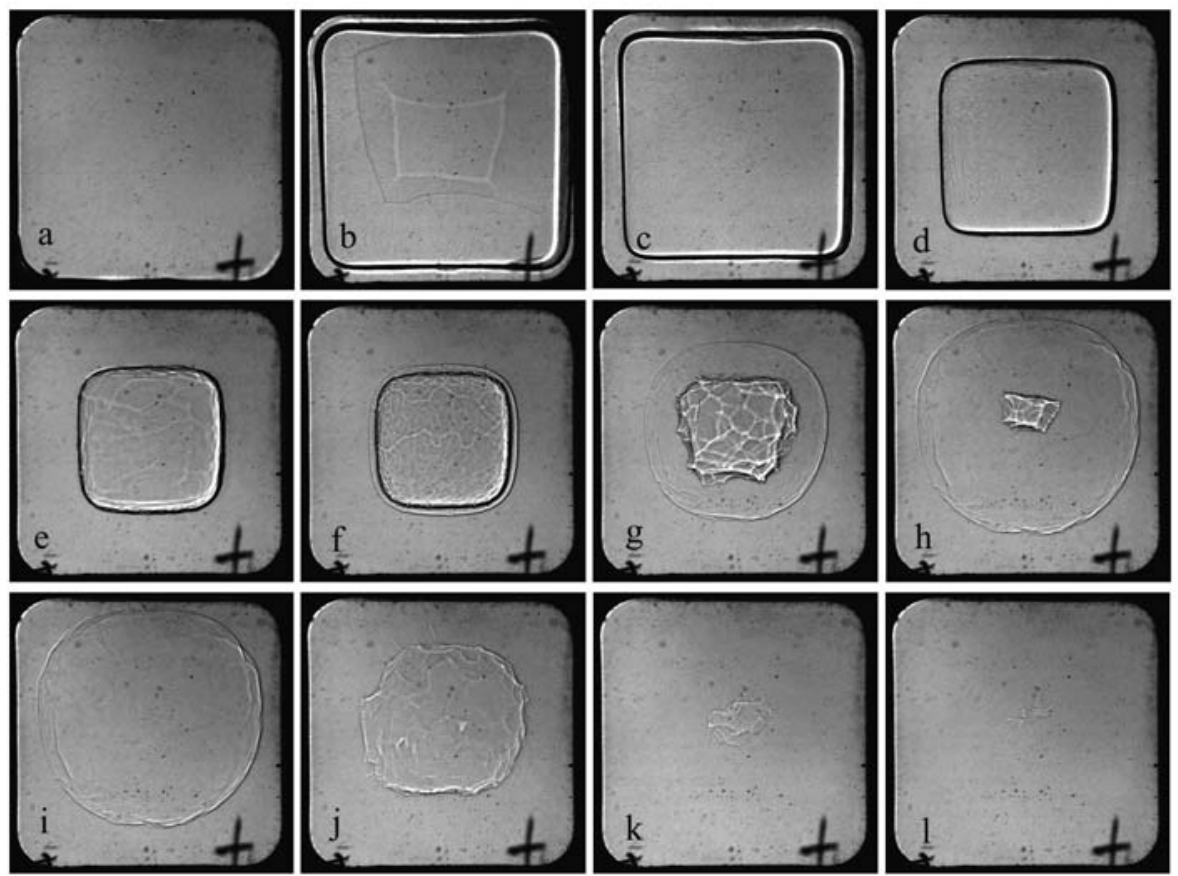

Fig. 4 Crystallization of the hcp solid through the bcc phase in the thick cell with the BCM (path B on the phase diagram in Fig. 2). Crystallization started at the bottom from the normal liquid at $2.36 \mathrm{~K}$ in the first image (a) and the hcp solid slowly grew along the melting curve of helium 4 during cooling down (b-e). The bcc solid appeared at the LS boundary at $1.77 \mathrm{~K}$ (f). As hcp was converted into bcc, the bcc region extended outwards with a disk geometry $(\mathbf{g}-\mathbf{i})$. At $1.66 \mathrm{~K}$, the superfluid was entirely frozen (i). Upon further cooling, the bcc region shrank (j and k) and finally disappeared (l) at $1.59 \mathrm{~K}$. The whole process took 3 hours. Two crosses carved on the windows at the lower corners help adjusting the focusing

from the melting curve. At this temperature, the solid phase is hcp and it grows from the cold walls as usual ( $4 \mathrm{~b}$ to $4 \mathrm{e}$ ). The white lines on Figs. $4 \mathrm{~b}$ to $4 \mathrm{e}$ are presumably grain boundaries in the solid layer which covers the windows (see Sect. 4). At 1.77 K, the bcc solid appears between the liquid in the central part and the hcp solid in the outer part. This is consistent with the temperature for the upper bcc-hcp transition $T_{\mathrm{u}}=1.772 \mathrm{~K}$ measured by Grilly [36] and by Hoffer et al. [28]. We conclude that the temperature gradient in the cell is small but not negligible since Figs. $4 \mathrm{f}$ to $4 \mathrm{~h}$ show the three phases (liquid, bcc and hcp from the center to the walls) when the copper walls are at $1.73 \mathrm{~K}, 1.69 \mathrm{~K}$, and $1.67 \mathrm{~K}$, respectively, definitely lower than the triple point at $T_{\mathrm{u}}$. We note that the bcc-liquid interface is much more irregular than the hcp-liquid interface (Figs. $4 \mathrm{~b}$ to $4 \mathrm{e}$ ). This is probably because the superfluid transition occurs at $1.76 \mathrm{~K}$ on the melting curve $[28,36]$, so that the liquid is now superfluid and the temperature gradient near the interface is much smaller; the surface tension becomes relevant and macroscopic grooves become visible where grain boundaries emerge at the liquid-solid interface. It also appears that the growth from the superfluid leads to larger grain sizes than from the normal liquid. Those grooves 
clearly indicate that the solid involves many GBs and grains. A detailed analysis of the GB grooves is given below in Sect. 4.2.

In Fig. $4 \mathrm{i}$ at $1.66 \mathrm{~K}$, all the liquid is frozen and only the bcc and hep phases remain. As the cell is further cooled down ( $4 \mathrm{j}$ to 41$)$, the hcp phase takes over and the bcc phase disappears. At the end at $1.59 \mathrm{~K}$ (1), 3 hours after Fig. 4a, only hcp solid remains. Here again, the crystallization seems to finish before it is expected $(1.59 \mathrm{~K}$ instead of $1.51 \mathrm{~K}$ ). The few black dots which are visible on these images are dust particles coming from the fill line, possibly some remainder of the flux used to solder it to the cell.

\subsection{Crystallization at Low Pressure: Path C}

Figure 5 shows crystallization along path $\mathrm{C}$ in the phase diagram (Fig. 2). This time, the cell was initially filled with liquid at $P=4.63 \mathrm{MPa}$ and $T=2.34 \mathrm{~K}$. Crystallization starts with the hcp phase at $2.19 \mathrm{~K}$ along the walls as usual (Fig. 5a). Figures $5 \mathrm{~b}$ and $5 \mathrm{c}$ show a polycrystalline film that progressively covers the window, which must be colder than the inside of the cell. Crystallization proceeds (5b to $5 \mathrm{~d}$ ) until the bcc phase appears (5e). Here again, when the liquid is superfluid, the interface shows many grooves due to grain boundaries emerging at the bcc-superfluid interface (Figs. 5g to 5k). The liquid becomes superfluid between Figs. $5 \mathrm{f}$ and $5 \mathrm{~g}$. The hcp phase disappears at $1.70 \mathrm{~K}$, between $5 \mathrm{~h}$ and $5 \mathrm{i}$. From $5 \mathrm{i}$ to $5 \mathrm{k}$ the bcc phase grows from the superfluid. At $1.56 \mathrm{~K}$ (Fig. 51), the cell is full of bcc solid helium 4. The next picture $(5 \mathrm{~m})$ is taken at $1.46 \mathrm{~K}$ where, due to the transformation of bcc into hcp which is denser, liquid reappears. Figure $5 \mathrm{n}$ shows that the liquid region has moved up due to the buoyancy force, because gravity is relevant when the temperature is sufficiently homogeneous in the solid sample. As the cell is cooled down further, the hcp phase grows from the superfluid (Fig. $5 \mathrm{~s}$ is taken at $1 \mathrm{~K}$ ). After 15 hours, the cell reaches $35 \mathrm{mK}$, but liquid drops are still present (Fig. 5t) because the initial pressure was not sufficiently high to crystallize the whole content of the cell.

\subsection{Melting Crystals Grown at Constant V}

We were able to melt the solid samples grown by the BCM by removing helium through the fill line. When the pressure approaches the melting pressure, sets of parallel lines appear on the windows (Fig. 6a). The typical distance between successive lines is $80 \mu \mathrm{m}$. It seems possible to us that the mechanism leading to the formation of these lines is the Asaro-Tiller-Grinfeld instability [37, 38]. It occurs at the surface of solid materials when a non-hydrostatic stress is applied. A corrugation appears which costs surface energy but decreases the elastic energy [19, 39, 40]. The threshold for this instability has been observed in helium 4 but at the liquid-solid interface where mass transport is easily achieved by the liquid phase [42-44]. Here, the modulation in the light intensity is a signature of a local density modulation, perhaps thin liquid regions where the liquid-solid interface unsticks from the window. For the formation of this corrugation, mass transport might be achieved by tiny liquid channels which exist where GBs meet the window (see below). If this interpretation is correct, one 


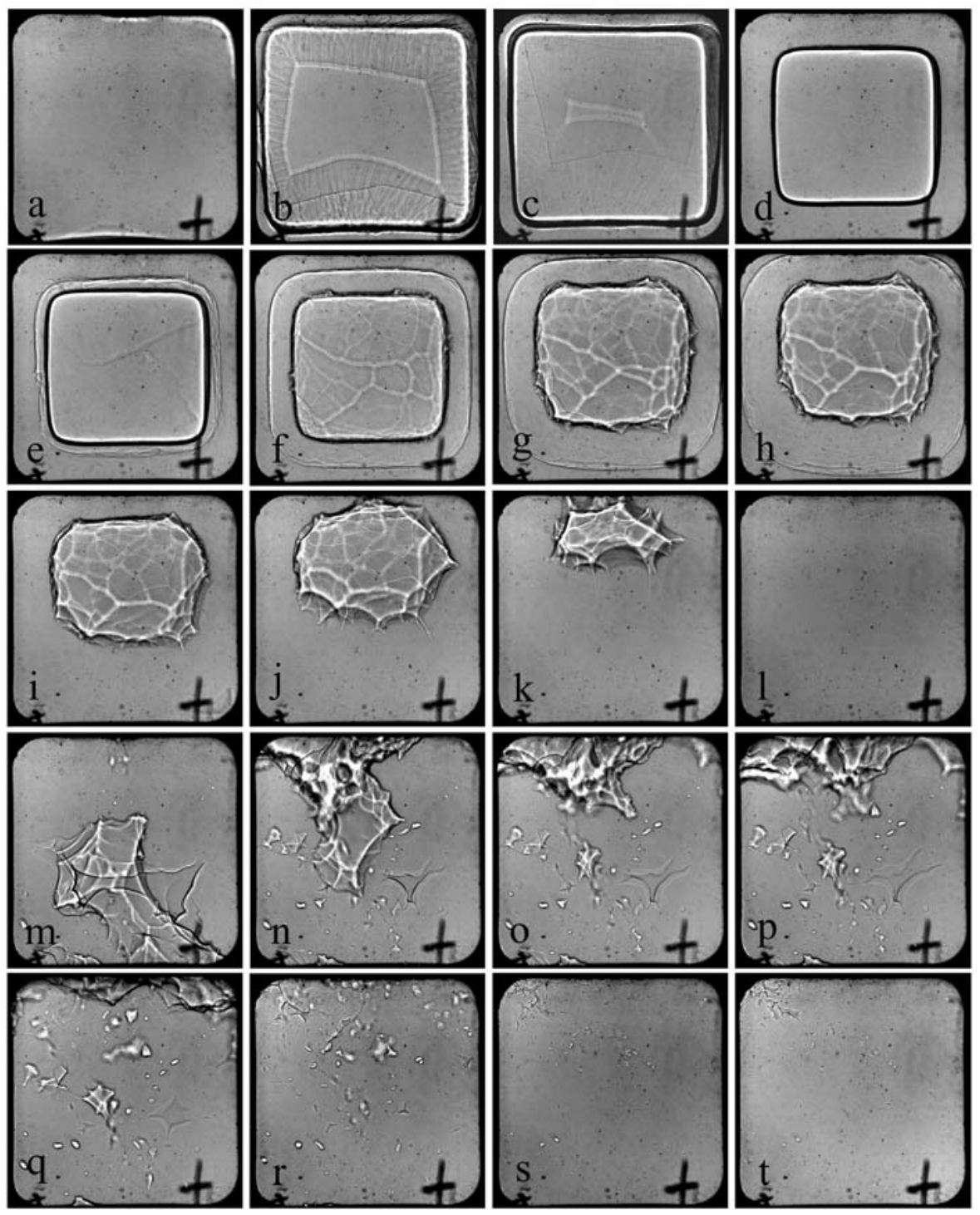

Fig. 5 Crystallization along path $\mathrm{C}$ of the phase diagram (see Fig. 2). Crystallization proceeds in the thick cell with the BCM through both the upper $(1.772 \mathrm{~K})$ and lower $(1.464 \mathrm{~K})$ transition points between hep and bcc on the melting curve of helium 4. Crystallization starts from the normal liquid at $2.19 \mathrm{~K}$ (a), and upon cooling, follows the meting curve of helium 4 as the hcp solid slowly grows (b to d). The bcc solid appears at the liquid-solid boundary, and some hcp transforms into bcc (e-h). The liquid becomes superfluid between (f) and (g). All hep is transformed in bcc at $1.70 \mathrm{~K}$ (i). Then the bcc solid grows into the superfluid region $(\mathbf{j}-\mathbf{k})$. The cell is full of bcc solid at $1.56 \mathrm{~K}$ (I). Suddenly, at $1.46 \mathrm{~K}$, bcc transforms into the denser hcp phase and liquid reappears at the bottom of the cell $(\mathbf{m})$. The liquid region rises within tens of seconds $(\mathbf{n}-\mathbf{p})$ due to buoyancy forces. The hcp solid keeps growing and the liquid region shrinks during cooling to $1 \mathrm{~K}(\mathbf{q}-\mathbf{s})$. When the cell reaches $35 \mathrm{mK}$ after 15 hours, some liquid drops remain in the hcp solid (t) 
Fig. 6 Melting of a solid sample grown by the BCM. The cell is slowly depressurized at low temperature (typically $35 \mathrm{mK}$ ) by removing helium through the fill line. As the pressure approaches the melting pressure $P_{\mathrm{m}}=2.53 \mathrm{MPa}$, sets of parallel lines appear on the window (a). These lines disappear when the pressure reaches $P_{\mathrm{m}}$ and small liquid droplets start coming out near the orifice of the fill line (b). Finally, thousands of liquid channels appear between tiny grains which ripen in a few seconds (c-d). After $18 \mathrm{~s}$, the grains have become macroscopic (e)

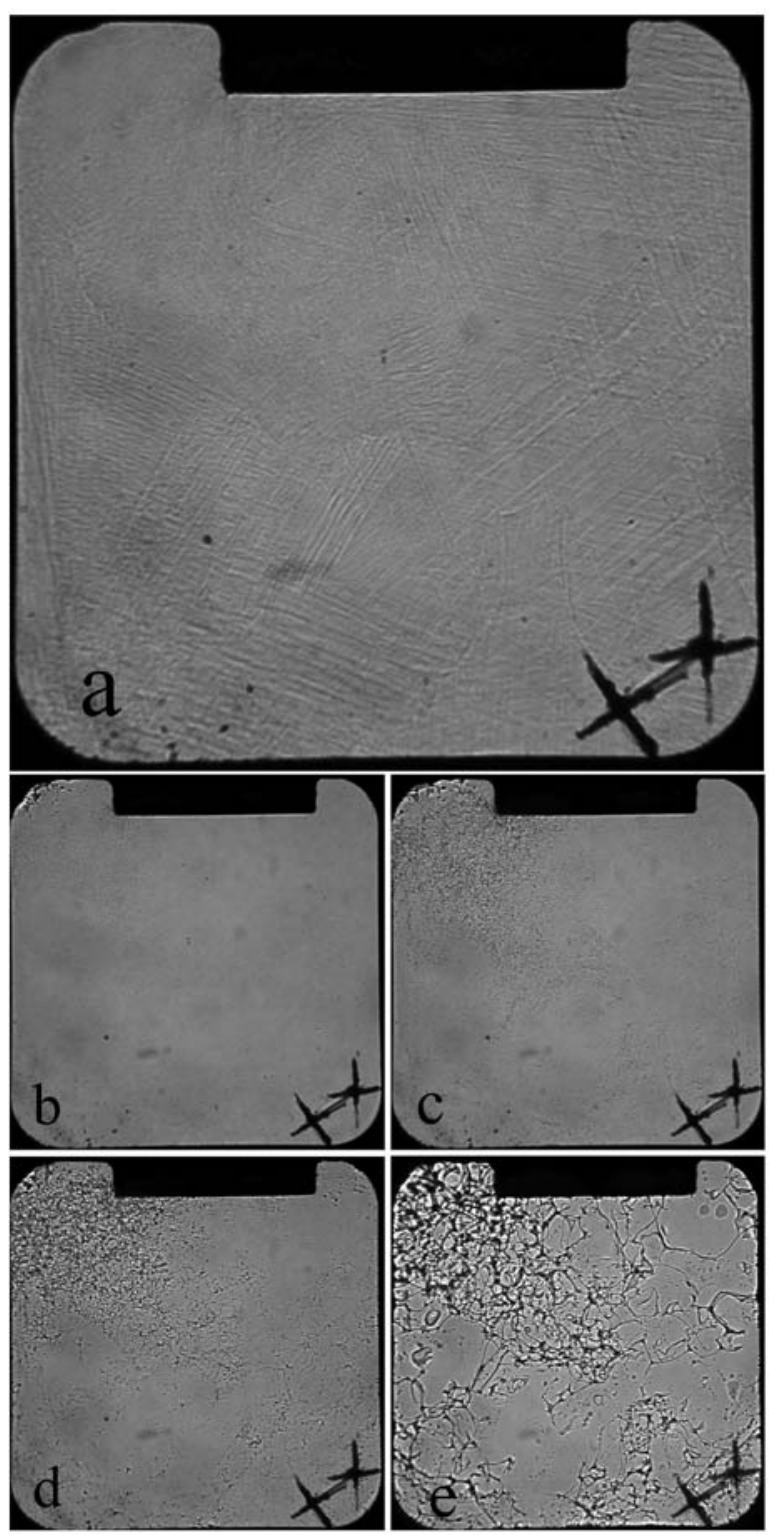

expects the minimum wavelength $\lambda_{\min }$ at which the surface is unstable to be related to the amplitude of the stress anisotropy $\delta \sigma$ through

$$
\lambda_{\min }=\frac{\pi E \gamma}{\delta \sigma^{2}}
$$

where $E \approx 30 \mathrm{MPa}$ is the Young modulus of solid helium 4 and $\gamma$ is the surface stiffness of the solid surface (typically $0.2 \mathrm{~mJ} / \mathrm{m}^{2}$ ) [19]. With these numerical values, and assuming that the selected wavelength is comparable to $\lambda_{\min }$, one finds a stress 
anisotropy $\delta \sigma \approx 15 \mathrm{kPa}$, which looks reasonable. When depressurizing a little more, the lines disappear, possibly because, very close to the liquid-solid equilibrium, stress inhomogeneities relax quickly, due to the existence of large liquid channels throughout the solid samples (see below).

When the liquid-solid equilibrium line is reached, a fine network of GBs shows up, starting from the fill line orifice (Fig. $6 \mathrm{~b}$ to e) and showing that it is indeed polycrystalline. What is seen on this figure is in fact a network of liquid channels at the contact of GBs with the windows. To understand why the contact lines of GBs with walls are liquid channels, we have measured the GB energy and the contact angle of the liquid-solid interface with walls. We shall come back to this in Sect. 4 below. Further depressurization leads to the appearance of grain boundaries everywhere, between grains whose size increases with time. This increase is a ripening effect due to surface tension. It is similar to what can be observed with a foam of soap bubbles: large crystals grow while small ones melt because the curvature of their surface is smaller.

The polycrystalline nature of solid samples grown at constant volume was already visible on Figs. 3 and 4 where the liquid-solid interface has a definite roughness, especially after a few millimeters of growth from the walls. We believe that temperature gradients in the cell decrease as solidification proceeds, so that capillary effects becomes relevant at the visible scale of micrometers. These capillary effects are mainly grooves due to the GB surface tension, as will be also shown in Sect. 4 .

We imagine that the polycrystalline nature of the solid is due to nucleation of crystals ahead of the crystallization front but this would need a systematic study. One could invoke the Asaro-Tiller-Grinfeld instability again [19, 37, 38, 41, 43, 44]. However, what we observe are grains with large differences in orientation, otherwise their boundaries would not produce liquid channels at their contact with the glass window (see Sect. 6). The Asaro-Tiller-Grinfeld instability leads to the formation of large concentrations of stresses but not to highly misoriented grains. There are other instabilities in directional solidification [45] but, once more, we do not see how they could lead to highly misoriented grains.

We have thus observed that solid samples grown by the BCM are polycrystalline with grain sizes of order $10 \mu \mathrm{m}$ or less. This is significantly smaller than $0.1 \mathrm{~mm}$, as assumed by Clark et al. $[17,46]$ who refer to thermal conductivity measurements $[47,48]$ and X-ray diffraction [51] in other experiments. Some recent X-ray measurements also showed large crystal grains $[49,50]$. It is possible that the density of GBs depends on the exact amplitude and shape of thermal gradients in the sample cell, which itself depends on the cell geometry, cell materials and cooling rate.

One could object that the depressurization itself breaks the solid samples in small crystallites. We cannot completely rule out this possibility but, if it were true, all the crystallites would have the same orientation and, in this case, no liquid channels would be present (see Sect. 4): it seems quite unlikely that, after the fracture, the small crystallites could rotate with respect to each other. We thus believe that the liquid channels reveal grains with large misorientations which have been formed during growth.

It is interesting to estimate which superfluid fraction could be built with the inside of GBs only, whatever their density. If, for simplicity, one considers grains forming a 
cubic lattice of period $l$, the ratio between surfaces of GBs and solid volume is $3 / l$. If each GB has an effective superfluid thickness $\alpha$, one needs:

$$
l=3 \alpha \frac{\rho_{\mathrm{S}}}{\rho_{\mathrm{S}}},
$$

where $\rho_{S}$ is the superfluid density and $\rho_{\mathrm{S}}$ is the total density of the solid. For $\rho_{S} / \rho_{\mathrm{S}}=$ $0.1 \%$ and if $\alpha$ is 0.1 to 1 interatomic distance $a=0.3 \mathrm{~nm}$, one finds a typical grain size $l=0.1$ to $1 \mu \mathrm{m}$. This is perhaps possible with samples grown by the BCM: when we observe grain sizes of order $10 \mu \mathrm{m}$ (see Fig. 6), some ripening may have already taken place, so that the grain size may have been smaller before melting. However, in order to explain supersolidity with GBs only, one would need to show that their superfluid transition temperature lies in the range 0.1 to $0.2 \mathrm{~K}$. In addition, we confirm that crystals grown slowly from the superfluid are usually single crystalline, as already known [19]. As a consequence, GBs cannot be responsible for the TO anomalies observed by Clark et al. [17] in samples grown from the superfluid at constant $T$ and $P$.

\subsection{Crystals Grown by Fast Injection}

To inject mass rapidly in the cell, we use the nitrogen trap situated on the fill line and separated from the cell by a valve. We first fill the trap with about $5 \mathrm{MPa}$ of helium 4, and then quickly open the valve for a fast mass injection in the cell. When this is done above the superfluid transition temperature $T_{\lambda}=1.76 \mathrm{~K}$, the growth is dendritic, as shown in Fig. 7 (top picture). Here the tangle of dendrites is very dense and there must be liquid regions inside the tangle, so that light is strongly scattered and the solid appears dark. Such dark helium samples have already been observed by Maekawa et al. [52] and by Ford et al. [53]. It is possible that the samples of Rittner and Reppy showing large NCRIF [9] are a dense tangle of dendrites since they have been solidified by a rapid temperature quench of the normal liquid as in the case of Fig. 7 (top picture). When the injection of normal liquid is not too fast, helium snow flakes in the shape of 6-fold symmetry stars may be seen (Fig. 7, bottom picture). Here again, the snow flakes look facetted in both the $c$ direction (they are flat) and in the six directions perpendicular to $c$. This is a further indication that facets may reappear at the surface of hcp helium crystals when their temperature is raised above about $2 \mathrm{~K}$ (see Sect. 3.1).

When mass is injected sufficiently fast at low temperature, one also observes polycrystals. Figure 8 shows crystallization in $140 \mathrm{~ms}$. At the end of the crystallization (Fig. 8f), the fill line blocked and we can assume that the final pressure in the cell was not far from the melting pressure $P_{\mathrm{m}}$. Slight inhomogeneities in the grey levels of this sample indicate a polycrystalline structure, especially on the enlarged picture $8 \mathrm{~g}$. To characterize the inside of such crystals, we have performed a simple light scattering experiment [27]. By shining a laser beam through the cell with an oblique incidence, we could separate the contribution from the windows. In some crystals, we could find light scattering from the interior of the solid, which is absent in the liquid or in a crystal grown slowly at constant volume. This shows that crystals grown by fast injection at low temperature may have inhomogeneities in density with sizes comparable to the wavelength of the laser $(600 \mathrm{~nm})$. Liquid channels a few micrometers wide near 
Fig. 7 Fast injection of mass in the normal liquid usually results in dendritic growth (top). At moderate injection rate, helium snow flakes show their 6-fold symmetry (bottom)

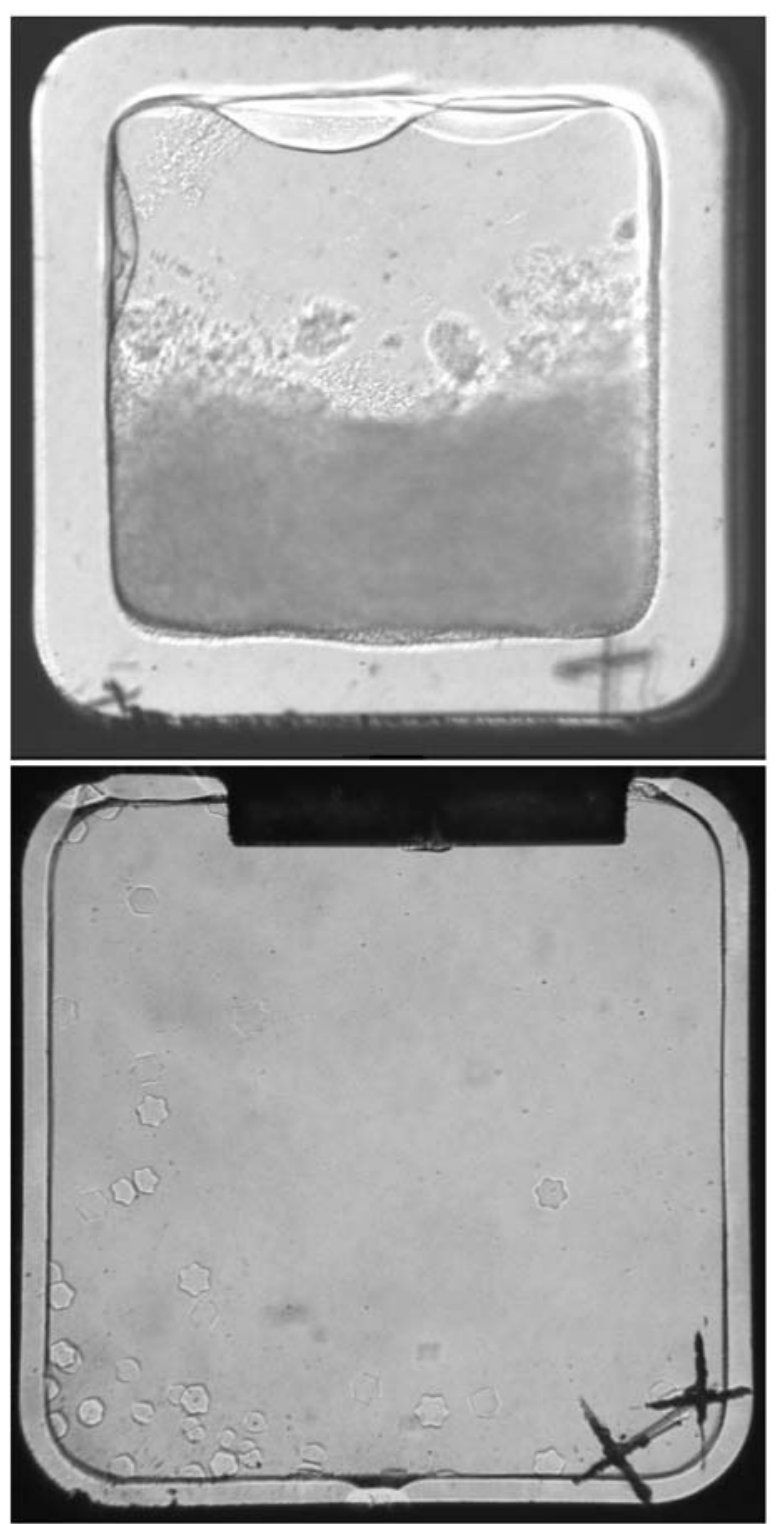

$P_{\mathrm{m}}$ can be found at the crossings of GBs, for the same reason as those we observed at the contact line of GBs with walls (see Sect. 6). The polycrystalline nature of the fast grown samples is even more clearly visible after a subsequent pressure release (Fig. 9): as in Fig. 6, the helium-window interface is invaded by a network of liquid channels, revealing the existence of grains whose size could be as small as one micrometer at the very beginning. The following images show that ripening takes place in a very short time when the liquid is superfluid, the grain size increasing quickly to millimeters in a time of order $100 \mathrm{~ms}$. 


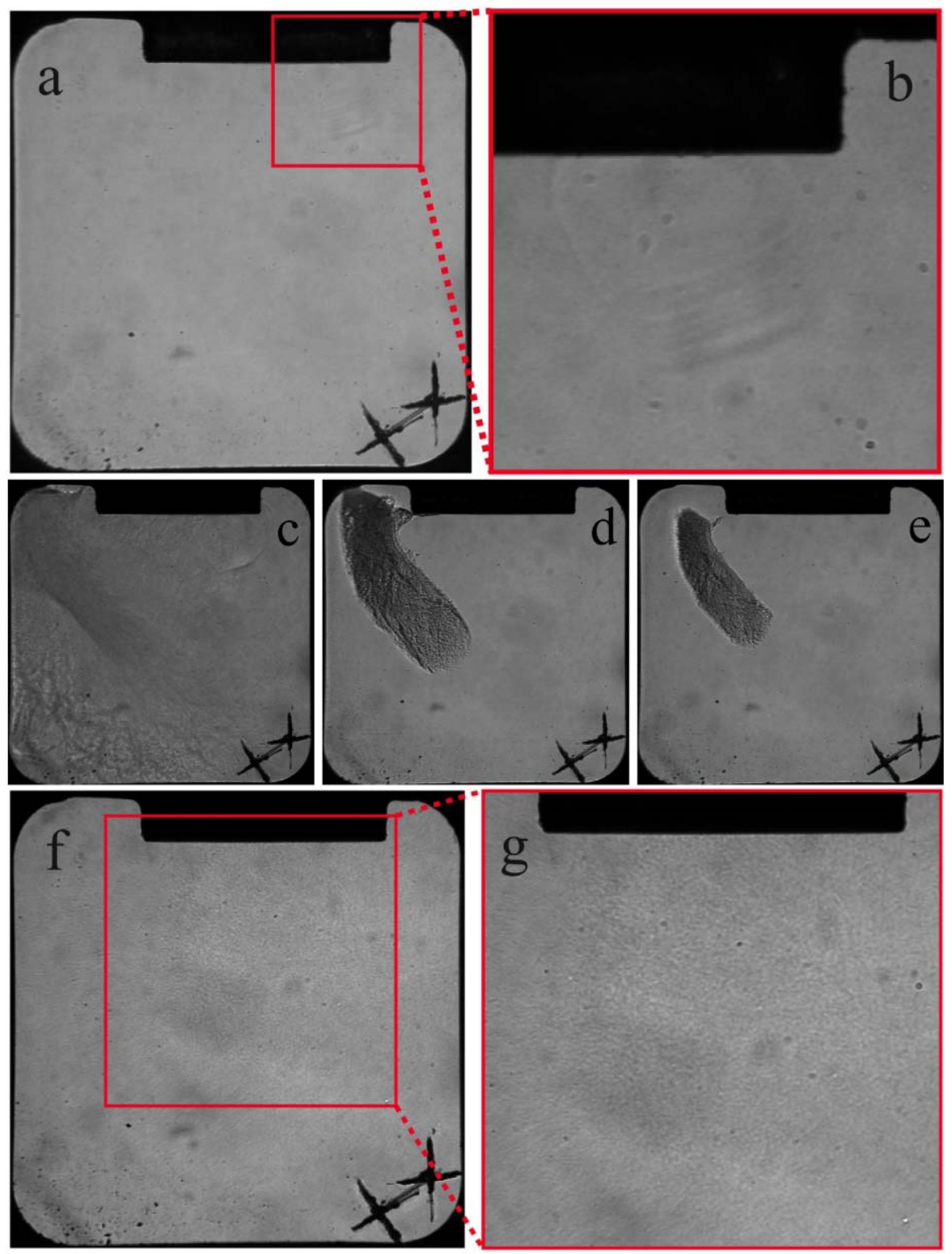

Fig. 8 (Color online) Crystallization by fast injection of mass in the superfluid. In this particular run, we injected mass quickly into the thin cell at $100 \mathrm{mK}$ (a), the first seed crystal nucleated and grew on the glass piece at the top of the cell (see enlarged picture in b). $70 \mathrm{~ms}$ after (a), a large number of crystals had already invaded the whole cell (c). Another $140 \mathrm{~ms}$ later (d, e), the cell was filled with a polycrystal whose grain size was small ( $\mathbf{g}$ is an enlargement of the top part of $\mathbf{f}$ ) 


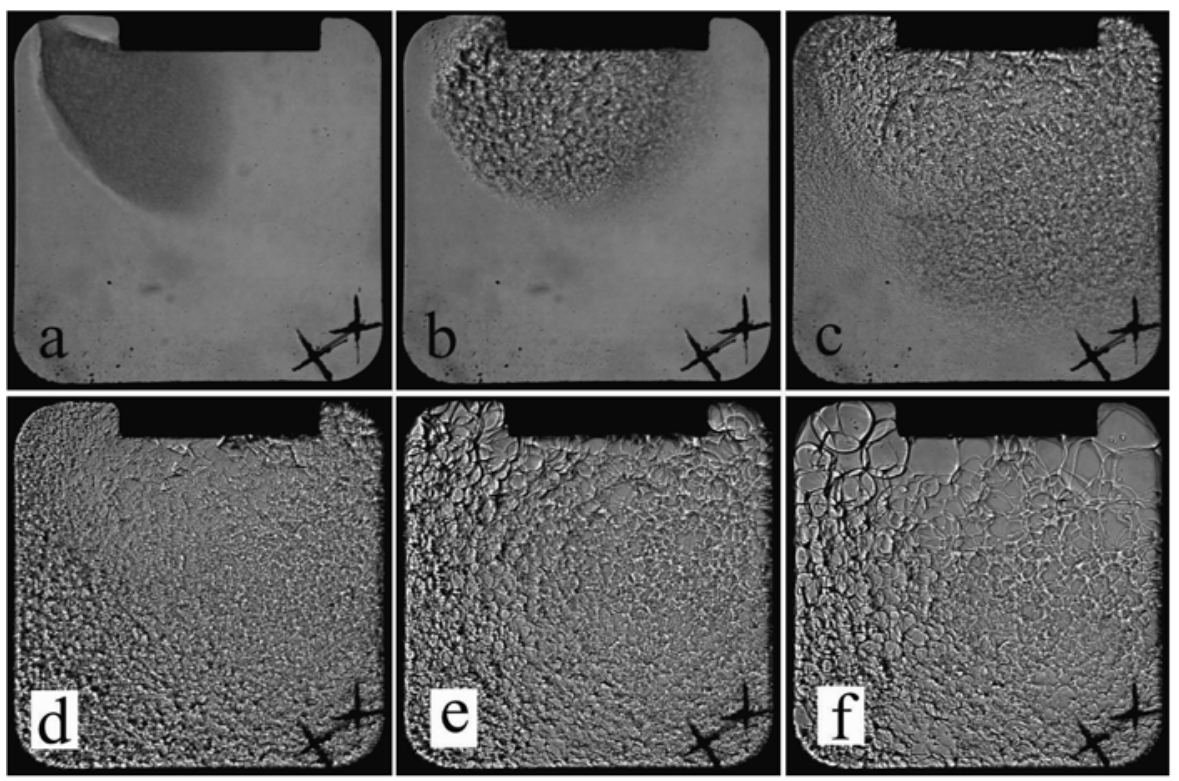

Fig. 9 Melting of solid grown by a fast injection of mass by slowly releasing the pressure in the cell at low temperature. The time interval between images (from a to d) is $90 \mathrm{~ms}$. Grains ripen gradually from e to $\mathbf{f}$

\section{Grain Boundaries}

\subsection{Preparation of Grain Boundaries}

Direct application of a small pressure step to the superfluid usually results in a single crystal (some exceptions are described below). In order to study single GBs, we use a different method which we describe now. We start either with a polycrystal grown by the $\mathrm{BCM}$ or by fast injection at low temperature, which saves time. We then remove helium from the cell through its fill line till a macroscopic liquid phase is visible. At that time, we stop removing helium and we observe ripening which proceeds quickly at the solid-liquid equilibrium. As already mentioned, this is a consequence of capillary effects with the liquid phase allowing fast mass transport from small crystals which melt to larger crystals which grow. In reality, the crystals which melt are those which are smaller than the capillary length

$$
l_{\mathrm{c}}=\sqrt{\frac{\sigma_{\mathrm{LS}}}{\left(\rho_{\mathrm{S}}-\rho_{\mathrm{L}}\right) g}} \simeq 1 \mathrm{~mm},
$$

so that their surface shows a definite curvature. In the above equation, $\sigma_{\mathrm{LS}}, \rho_{\mathrm{S}}, \rho_{\mathrm{L}}$ and $g$ are the solid-liquid interfacial energy, the solid- and the liquid-density, and the acceleration of gravity, respectively.

One is usually left with a few crystals larger than $l_{\mathrm{c}}$. With a thin cell, the GBs are oriented nearly perpendicular to the windows. This occurs for capillary reasons, 
like in a soap foam between two plates: interfaces perpendicular to the walls have a smaller area. In order to keep only two grains, one has to remove some more helium. If this is done slowly enough, the smallest crystals melt one after the other till only two are left. At this stage, we can grow them again, to form a large bi-crystal with only one GB, which is rather stable in time (see Fig. 10). We can align the GB close to the vertical direction using successive growth and melting cycles. If needed, we can finally adjust the optical axis precisely parallel to the GB plane by moving the camera.

\subsection{The Grain Boundary Energy}

Figure 10 shows three examples of bi-crystals. The pictures b, d, f show growth shapes corresponding respectively to the equilibrium shapes a, c, e. The growth shapes reveal facets, and one can see that the pictures a, b correspond to a GB between two grains with rather different orientations. The picture $g$ is an enlargement of the GB seen on picture a.

As explained in [12], GBs emerging at the liquid-solid interface make a groove to reach the mechanical balance between the GB surface tension $\sigma_{\mathrm{GB}}$ and the liquidsolid interfacial tension $\sigma_{\mathrm{LS}}$. In a first approximation, $\sigma_{\mathrm{GB}}=2 \sigma_{\mathrm{LS}} \cos \theta$ where $2 \theta$ is the dihedral angle of the groove. However, since the two crystals have different orientations, the liquid-solid interface makes an angle $\theta_{1}$ with the vertical on the left (crystal 1) and an angle $\theta_{2}$ on the right (see Fig. 11). As a consequence, a more rigorous expression is

$$
\sigma_{\mathrm{GB}}=\sigma_{1} \cos \theta_{1}+\sigma_{2} \cos \theta_{2} .
$$

Furthermore, since the GB is not strictly vertical, the above equation is slightly incorrect with $\theta_{1,2}$ measured from the vertical. However, we have verified that the corresponding error on the measurement of $\sigma_{\mathrm{GB}}$ is around $0.5 \%$, that is small compared to the final error bar on our measurements $( \pm 2 \%)$. Our definition of angles and (5) can be kept for simplicity and we may still define the dihedral angle $2 \theta=\theta_{1}+\theta_{2}$.

For three examples of crystals like (10a), the groove is deep with sharp but nonzero angles (see below). When trying to grow the solid by directly pressurizing the superfluid, one usually ends with a single crystal. However, it can happen that two crystals with similar orientations are produced (Figs. 10c, d, e, f). They show a very shallow groove, indicating a GB with small $\sigma_{\mathrm{GB}}$, perhaps a stacking fault. This observation is consistent with the recent measurements by Junes et al. [54].

In order to make a precise measurement of the groove angles, we use the following procedure. We analyze the gray levels of the image over successive vertical lines, taking for each line the liquid-solid interface position at the darkest pixel. Then, on each side of the groove, we fit the liquid-solid interface line with the Laplace equation using the surface tension and the angle as adjustable parameters. We have assumed that the curvature in the plane perpendicular to the windows was approximately constant, so that we could use a simplified version of the Laplace equation in the following form $[55,56]$ :

$$
x=x_{0}+l_{\mathrm{c}} \arg \cosh \left(\frac{2 l_{\mathrm{c}}}{z}\right)-2 l_{\mathrm{c}} \sqrt{1-\frac{z^{2}}{4 l_{\mathrm{c}}^{2}}} .
$$



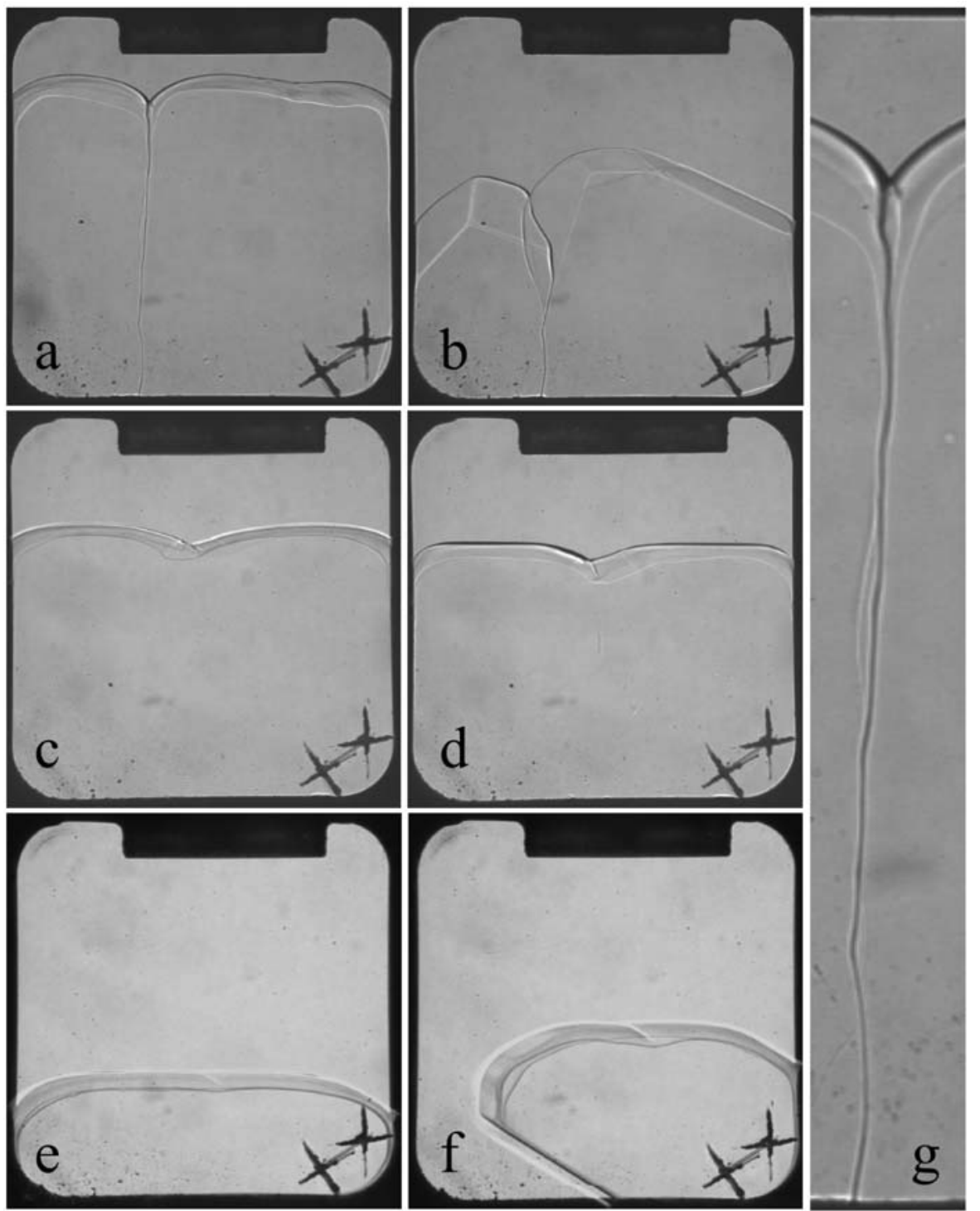

Fig. 10 Three pairs of images showing equilibrium shapes (a, c, e) together with growth shapes which reveal the crystal orientation $(\mathbf{b}, \mathbf{d}, \mathbf{f})$. When the two crystal grains have a large difference in orientation (a, b), their boundary ends as a deep groove at the liquid-solid interface. A zoom of (a) shows that the contact lines of the GB with the windows are in fact liquid channels (g). Crystals with similar orientations can be obtained by direct growth $(\mathbf{c}, \mathbf{d}, \mathbf{e}, \mathbf{f})$. In this case, the groove is shallow with no liquid channels on the windows. Two crosses carved on the windows help adjusting the focusing. Reprinted Fig. 1 with permission from S. Sasaki, F. Caupin, S. Balibar, Phys. Rev. Lett. 99, 205302 (2007) [12]. Copyright (2007) by the American Physical Society 
Fig. 11 The dihedral angle $2 \theta=\theta_{1}+\theta_{2}$ is determined by fitting each crystal profile with the Laplace equation near the groove

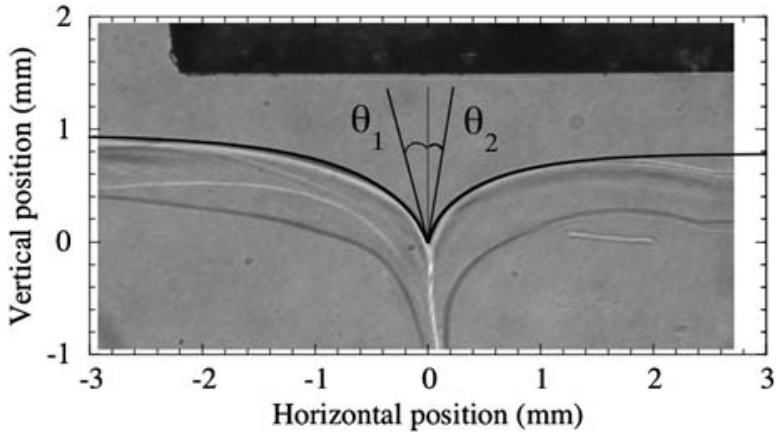

Table 1 The dihedral angle $2 \theta=\theta_{1}+\theta_{2}$ of grain boundaries, as measured with three different samples (S1, S2, S3)

\begin{tabular}{|c|c|c|c|c|c|c|}
\hline \multirow[t]{2}{*}{ Sample } & \multicolumn{3}{|c|}{ Angles $\left({ }^{\circ}\right)$} & \multicolumn{3}{|c|}{ Surface tensions $\left(\mathrm{mJ} \mathrm{m}^{-2}\right)$} \\
\hline & $\theta_{1}$ & $\theta_{2}$ & $2 \theta$ & $\sigma_{1}$ & $\sigma_{2}$ & $\sigma_{\mathrm{GB}}$ \\
\hline S1 & 20.3 & 1.8 & 22.1 & 0.113 & 0.0800 & 0.186 \\
\hline $\mathrm{S} 2$ & 20.0 & 12.4 & 32.4 & 0.0985 & 0.0674 & 0.158 \\
\hline S3 & 14.8 & 14.2 & 29 & 0.0555 & 0.0823 & 0.133 \\
\hline
\end{tabular}

Since the surface tension is known to be anisotropic for hcp helium 4 crystals [19], the fit was made on a small part of the meniscus (about $2.5 \mathrm{~mm}$ long) on each side of the groove. Figure 11 shows that the result is close to the real meniscus even far away from the groove, meaning that, in this particular case, the anisotropy of the surface stiffness was not very large. Table 1 gives the results corresponding to three pairs of crystals (S1, S2 and S3).

Care was taken to view the groove along the GB direction: the optical axis was rotated until the lowest $2 \theta$ was found. The best fit gave slightly different values of $\sigma_{\mathrm{LS}}$ and $\theta$ on each side of the GB (Table 1). We have found that the dihedral angle of the groove $\left(\theta_{1}+\theta_{2}\right)$ is strictly positive, of order $30^{\circ}$. The GB energy is of order $0.15 \mathrm{~mJ} \mathrm{~m}^{-2}$, strictly less than $\sigma_{1}+\sigma_{2}$. Note that, for our three samples, we have values for $\sigma_{1,2}$ which are low compared to the average surface tension $\sigma_{\mathrm{LS}}=0.17 \mathrm{~mJ} \mathrm{~m}^{-2}$ [19]. This might be a consequence of our use of isotropic surface tensions $\sigma_{1,2}$. A rigorous treatment of the anisotropy would need to replace $\sigma \cos \theta$ terms in (5) by $\sigma \cos \theta+(\partial \sigma / \partial \theta) \sin \theta$ terms. One should also consider the possible variation as a function of height of the curvature in a plane perpendicular to the windows. But such more rigorous expressions would introduce too many parameters in our fits. As we are primarily interested in measuring the dihedral angle, the above procedure is sufficient. The good quality of the fit away from the groove explains why the use of an integrated version of (6)

$$
\left(\rho_{\mathrm{S}}-\rho_{\mathrm{L}}\right) g(\Delta z)^{2}=2 \sigma_{\mathrm{LS}}(1-\sin \theta)
$$

gives similar values for $\theta$ ( $\Delta z$ is the depth of the groove). Equation (7) is similar to one widely use to determine the liquid-solid surface tension of classical systems, in experiments where gravity is replaced by a temperature gradient $[57,58]$. 
The physical meaning of our measurements is that, even at the liquid-solid equilibrium, a GB is not completely wet by the liquid phase. It remains a thin layer. This is consistent with the simulations by Pollet et al. [13, 14], who showed that GBs are generally made of three disordered layers of atoms. Complete wetting $(\theta=0)$ would imply that the GB is invaded by a macroscopic thickness of liquid, in which case it would be simply made of two liquid-solid interfaces and $\sigma_{\mathrm{GB}}$ would be equal to $2 \sigma_{\mathrm{LS}}$. Note that the wetting of GBs is an important issue in materials science. It has even been predicted that GBs could premelt [59], that is melt before the bulk, being invaded by a thick liquid layer at a temperature below the bulk melting temperature $T_{\mathrm{m}}$. However, experimental evidence is rather difficult to obtain. Hsieh and Balluffi studied aluminum films, and concluded that "no complete grain boundary melting was detectable by local diffraction contrast at temperature lower than $0.999 T_{\mathrm{m}}$ " [60]. GB premelting has been reported in colloidal crystals [61]. In fact, although premelting is possible in systems with short range forces, it cannot happen in the case of long range forces (e.g. van der Waals forces) [62-64]. For more details we refer to [65].

Franck et al. $[66,67]$ studied thin films (less than $50 \mu \mathrm{m}$ thick) of fcc helium at high pressure and temperature. Their observation technique used a Schlieren method which did not allow them to measure precisely the dihedral angle; they were only able to state that $0 \leq 2 \theta \leq 30^{\circ}$. Their experiment is sometimes erroneously interpreted as evidence for complete wetting [68, 69]. In fact, because the films lie on a sapphire substrate favorable to the liquid, even if the solid grains detach from each other, it may be due to the growth of liquid channels between the grains on the sapphire, rather than to GB melting.

\section{The Contact Angle of the Liquid-Solid Interface with Walls}

Our experimental setup allows us to measure the contact angle of the liquid-solid interface with various walls. For convenience, we measure it on the liquid side. This angle was already known as being equal to about $45^{\circ}$ [19]. However, it was never systematically studied. Here, we present measurements for copper, glass and graphite walls. As we shall see, this angle depends weakly on the nature of the wall but it is hysteretic: it depends on whether the interface recedes or advances, and also on the velocity.

The contact angle is determined by fitting the liquid-solid interface profile with the Laplace equation (6) (see Figs. 12 and 13), using the procedure described in Sect. 4.2.

We have observed that the advancing contact angle $\theta_{\text {adv }}$ (obtained for slow crystallization, see Figs. 12a and 13a) is smaller than the receding contact angle $\theta_{\text {rec }}$ (obtained for slow melting, see Figs. 12b and 13b). Table 2 summarizes angle measurements for 6 different samples at a typical velocity of $10 \mu \mathrm{m} \mathrm{s}^{-1}$. The samples have different orientations and the angles change, but $\theta_{\text {adv }}$ is always smaller than $\theta_{\text {rec }}$. One can summarize all the results with the average values $\theta_{\mathrm{adv}} \simeq 22^{\circ}$ and $\theta_{\text {rec }} \simeq 55^{\circ}$. However, one should keep in mind that the angle values are not randomly scattered: they depend on the crystal orientation, so that it would be meaningless to give an error bar on the average values above. 


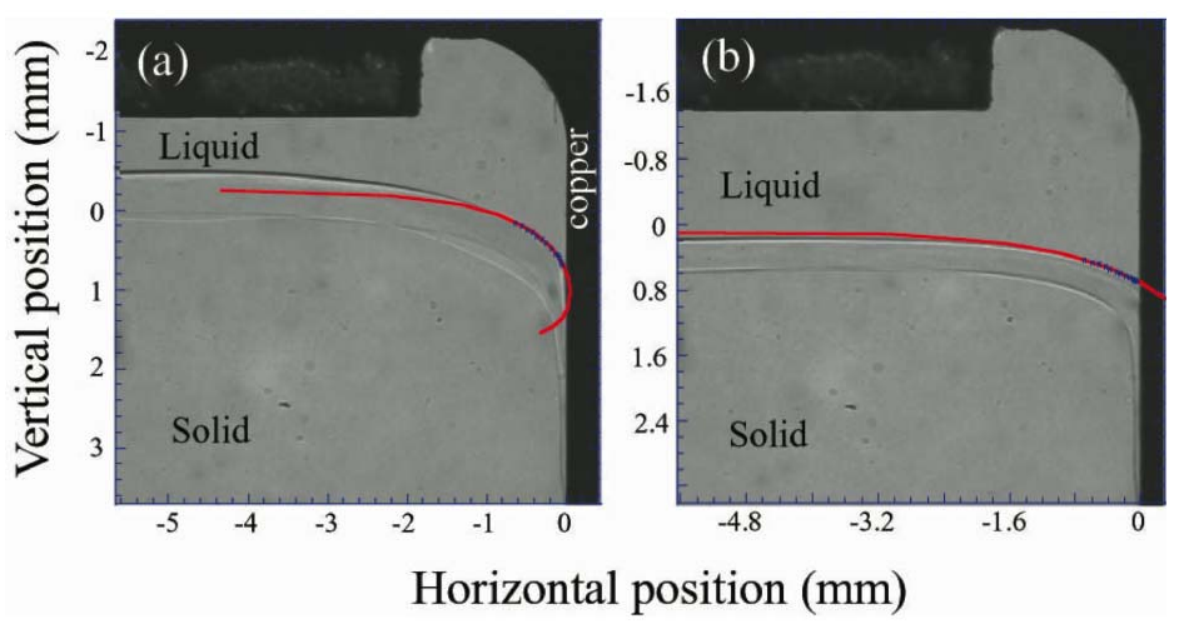

Fig. 12 (Color online) Contact of a crystal (sample S4) with a copper wall at $65 \mathrm{mK}$ : (a) for slow growth at $60 \mu \mathrm{m} \mathrm{s}^{-1}$, and (b) for slow melting at $5 \mu \mathrm{m} \mathrm{s}^{-1}$. Superimposed on the photographs are solid lines corresponding to the Laplace equation (6)

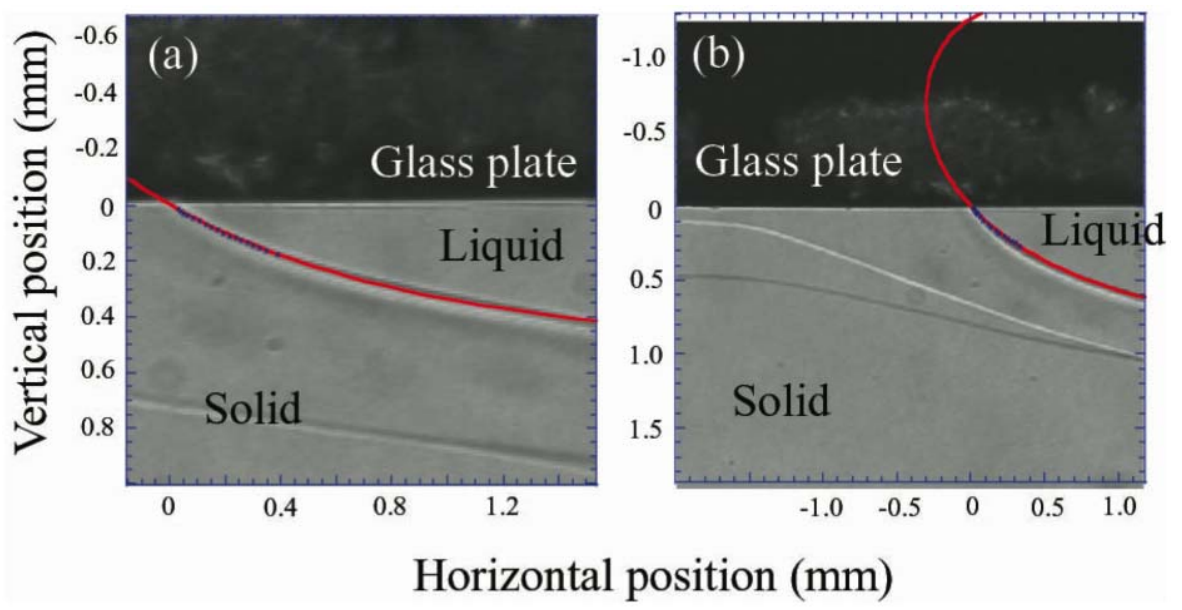

Fig. 13 (Color online) Contact of a crystal (sample S4) with a glass plate at $70 \mathrm{mK}$ : (a) for slow growth at $16 \mu \mathrm{m} \mathrm{s}^{-1}$, and (b) for slow melting at $10 \mu \mathrm{m} \mathrm{s}^{-1}$. Superimposed on the photographs are solid lines corresponding to the Laplace equation (6)

As for the contact with a glass wall, we have observed the same type of behavior, as summarized in Table 3. Apparently, the advancing angle is about $36^{\circ}$ and the receding angle about $51^{\circ}$. If the difference is significant, it shows a slightly smaller hysteresis for glass than for copper. This result would be consistent with the generally accepted idea that the hysteresis is an increasing function of roughness [70], since the copper walls are not polished after machining.

Clean graphite surfaces are known to favor the epitaxial growth of hcp helium 4 crystals, and we have tried to nucleate crystals with graphite substrates. For this, we 
Table 2 Even for slow motion $(\approx 10 \mu \mathrm{m} / \mathrm{s})$ of the liquid-solid interface, its contact angle with a copper wall is hysteretic.

During growth the angle $\theta_{\mathrm{adv}}$ is smaller than during melting $\left(\theta_{\text {rec }}\right)$. Missing data correspond to cases where the angle measurement was made difficult by the presence of a facet near the contact line

\begin{tabular}{rll}
\hline & $\theta_{\text {adv }}$ & $\theta_{\text {rec }}$ \\
\hline S4 & $22 \pm 2^{\circ}$ & $61 \pm 2^{\circ}$ \\
S5 & $26 \pm 4^{\circ}$ & - \\
S7 & $14 \pm 7^{\circ}$ & $60 \pm 1^{\circ}$ \\
S8 & - & $57 \pm 2^{\circ}$ \\
S10 & - & $48 \pm 6^{\circ}$ \\
S11 & $26 \pm 4^{\circ}$ & $50 \pm 2^{\circ}$ \\
\hline
\end{tabular}

Table 3 Hysteresis of the contact angle on a glass wall. The results are similar to the case of a copper wall (Table 2). Here again, the presence of facets sometimes made the measurement impossible

\begin{tabular}{lll}
\hline & $\theta_{\mathrm{adv}}$ & $\theta_{\mathrm{rec}}$ \\
\hline S4 & $30 \pm 3^{\circ}$ & $47 \pm 6^{\circ}$ \\
S5 & $30 \pm 8^{\circ}$ & $49 \pm 3^{\circ}$ \\
S6 & - & $53 \pm 2^{\circ}$ \\
S7 & $14 \pm 1^{\circ}$ & $49 \pm 4^{\circ}$ \\
S8 & $33 \pm 5^{\circ}$ & $54 \pm 6^{\circ}$ \\
S9 & $23 \pm 7^{\circ}$ & $61 \pm 5^{\circ}$ \\
S10 & - & $51 \pm 4^{\circ}$ \\
S11 & - & $54 \pm 6^{\circ}$ \\
S12 & - & $51 \pm 4^{\circ}$ \\
S13 & $28 \pm 2^{\circ}$ & $44 \pm 4^{\circ}$ \\
\hline
\end{tabular}

have glued highly oriented pyrolytic graphite (HOPG) and papyex pieces in the cell. The pieces were freshly cleaved but exposed to air during the mounting of the cell. After closure the cell was warmed up to $100^{\circ} \mathrm{C}$ and flushed a dozen of times with clean helium gas. This was not sufficient to obtain crystal nucleation on the graphite. In the old experiment by Balibar and Castaing [71], the graphite was taken to high temperature (about $1000 \mathrm{~K}$ ) under vacuum by passing a high current through it. In the present cell, this could not be done. We measured contact angles on these partially cleaned graphite pieces which were similar to what we found on copper and on glass: the advancing angle was between $31^{\circ}$ and $43^{\circ}$ and the receding angle between $44^{\circ}$ and $62^{\circ}$. It appeared better to pump for three days on the cell at $100^{\circ} \mathrm{C}$ without flushing, in which case we observed nucleation of oriented crystals on the graphite pieces (see Fig. 14). However, the nucleation did not always take place there, indicating that the degassing was probably not yet sufficient. As for the contact angle on graphite it was again of the order of $45^{\circ}$, except of course when the crystal orientation was parallel to that of the graphite. In fact, when helium crystals are not oriented parallel to the graphite, and since the graphite surface is probably covered by a thin solid helium layer, one expects the contact angle of the liquid-solid interface to be comparable to the above mentioned groove angle of the grain boundary between two misoriented crystals, i.e. $30^{\circ}$. 
Fig. 14 Nucleation of a helium crystal on a graphite piece. In this particular case, several pieces of graphite have been glued to the walls of the cell: two large rectangular pieces of HOPG graphite (back window and left wall), two smaller HOPG pieces (bottom), and two papyex pieces (top and right-wall). The orientation of the hcp helium crystal is parallel to that of the graphite on the bottom right piece $(c$-helium facets are parallel to the surface of the graphite)
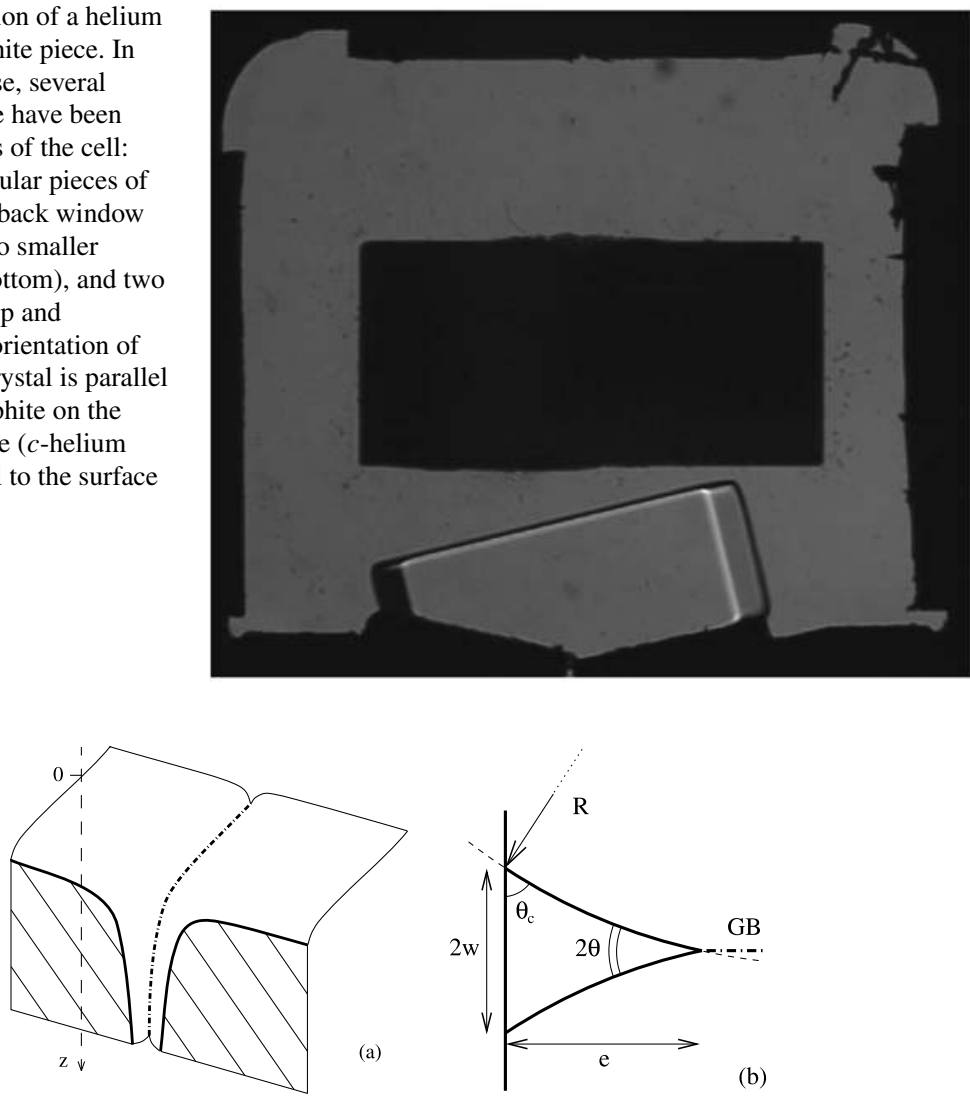

Fig. 15 (a) Three dimensional view of the contact between a grain boundary (dash-dotted line) and a wall. The hatched area shows the contact of the wall with the solid. (b) Horizontal cross section of the liquid channel near the wall. Reprinted Fig. 3 with permission from S. Sasaki, F. Caupin, S. Balibar, Phys. Rev. Lett. 99, 205302 (2007) [12]. Copyright (2007) by the American Physical Society

\section{Contact Lines of Grain Boundaries}

\subsection{Liquid Channels along Walls}

As shown on Figs. 10a, b, g, a liquid channel can exist at the contact line between the GB and the glass window of the cell. As explained in [12], this phenomenon is due to the glass wall being preferentially wet, although not completely, by the liquid phase. It occurs when the GB energy $\sigma_{\mathrm{GB}}$ is large enough. Here we give more details on the model introduced in [12].

Figure 15a shows a schematic view of a liquid channel. In the present treatment we neglect elasticity, and assume the surface tensions to be isotropic. Gravity and the force balance on the contact line between the liquid and GB then require the GB to lie in a vertical plane perpendicular to the wall, and the liquid channel to be symmetric with respect to this plane. Let $\theta_{\mathrm{c}}$ and $2 \theta$ be the contact angle of the liquidsolid (LS) interface on the wall, and the opening angle at the liquid-GB contact line, 
respectively. We have

$$
\cos \theta_{\mathrm{c}}=\frac{\sigma_{\mathrm{SW}}-\sigma_{\mathrm{LW}}}{\sigma_{\mathrm{LS}}} \text { and } \cos \theta=\frac{\sigma_{\mathrm{GB}}}{2 \sigma_{\mathrm{LS}}}
$$

where $\sigma_{\mathrm{LS}}, \sigma_{\mathrm{SW}}, \sigma_{\mathrm{LW}}$, and $\sigma_{\mathrm{GB}}$ the liquid-solid, solid-wall, liquid-wall, and GB surface tensions, respectively.

$e$ and $2 w$ are the maximum thickness and width of the liquid channel, and $R$ the horizontal radius of curvature of the liquid-solid interface (see Fig. 15b). Trigonometry gives the relations:

$$
e=R\left(\cos \theta_{\mathrm{c}}-\sin \theta\right)
$$

and

$$
w=R\left(\cos \theta-\sin \theta_{\mathrm{c}}\right) .
$$

We can also calculate the cross section of the channel:

$$
\Sigma=R^{2}\left[\left(\cos \theta_{\mathrm{c}}-\sin \theta\right)\left(\cos \theta-\sin \theta_{\mathrm{c}}\right)+\cos \left(\theta+\theta_{\mathrm{c}}\right)+\theta+\theta_{\mathrm{c}}-\frac{\pi}{2}\right] .
$$

Our aim is to compute $e$ and $w$ as a function of the depth $z$. We take $z=0$ at the flat liquid-solid interface at the top of the grains, where the pressure is the equilibrium pressure $P_{\text {eq }}$ (see Fig. 15a).

Hydrostatic equilibrium in the liquid implies that the liquid pressure is $P_{\mathrm{L}}(z)=$ $P_{\text {eq }}+\rho_{\mathrm{L}} g z$, with $\rho_{\mathrm{i}}$ the mass per unit volume of the phase $\mathrm{i}(\mathrm{i}=\mathrm{L}$ for liquid, $\mathrm{S}$ for solid), and $g$ the gravity.

The chemical potential must be uniform:

$$
\begin{aligned}
\mu_{\mathrm{L}}\left[P_{\mathrm{L}}(z)\right]-g z & =\mu_{\mathrm{L}}\left[P_{\mathrm{eq}}\right]=\mu_{\mathrm{S}}\left[P_{\mathrm{S}}(z)\right]-g z \\
& =\mu_{\mathrm{S}}\left[P_{\mathrm{eq}}\right]+\frac{P_{\mathrm{S}}(z)-P_{\mathrm{eq}}}{\rho_{\mathrm{S}}}-g z .
\end{aligned}
$$

As $\mu_{\mathrm{S}}\left[P_{\mathrm{eq}}\right]=\mu_{\mathrm{L}}\left[P_{\mathrm{eq}}\right]=\mu_{\mathrm{eq}}$, this implies hydrostatic equilibrium in both phases: $P_{\mathrm{L}}(z)=P_{\mathrm{eq}}+\rho_{\mathrm{L}} g z$ and $P_{\mathrm{S}}(z)=P_{\mathrm{eq}}+\rho_{\mathrm{S}} g z$. The pressure difference through the LS interface sets its curvature. At depths large enough compared to the capillary length $l_{\mathrm{c}}$, the curvature in the horizontal plane dominates, and the LS interfaces in a horizontal plane are circular arcs of radius $R=\sigma_{\mathrm{LS}} /\left[P_{\mathrm{S}}(z)-P_{\mathrm{L}}(z)\right]=l_{\mathrm{c}}^{2} / z$, with the liquid on the convex side.

The liquid channel exists if and only if $e$ and $w$ are positive, that is $\theta_{\mathrm{c}}<\pi / 2$, $\theta<\pi / 2$, and $\theta+\theta_{\mathrm{c}}<\pi / 2$. It is easy to see from Fig. $15 \mathrm{~b}$ that these are the conditions required to construct an arc with the correct contact angles, and the liquid on the convex side.

Another approach is to consider two grains touching each other and the wall, in contact with a thermostat at $T$ and a particle reservoir at $\mu_{\text {eq. }}$. To simplify the discussion, we now consider a horizontal channel of length $\ell$ at a fixed depth $z$. The relevant thermodynamical potential is the grand potential $\Omega=F-\mu_{\mathrm{eq}} N$, where $F$ is the free energy and $N$ the number of particles. If the angles $\theta$ and $\theta_{\mathrm{c}}$ are fixed, the 
section shown in Fig. 15b is fully determined by the value of $R$. To find the equilibrium shape, one has to minimize $\Omega$. For a given $R$, it implies $\mu_{\mathrm{L}}=\mu_{\mathrm{C}}=\mu_{\mathrm{eq}}$, so that $P_{\mathrm{L}}(z)=P_{\mathrm{eq}}+\rho_{\mathrm{L}} g z$ and $P_{\mathrm{S}}(z)=P_{\mathrm{eq}}+\rho_{\mathrm{S}} g z$ (see above). The variation of $\Omega$ between the initial situation where the two grains touch the wall everywhere $(R=0)$, and the final one with a channel for a given $R$, is

$$
\Delta \Omega=\ell \Sigma\left(P_{\mathrm{S}}-P_{\mathrm{L}}\right)+\Delta E=\ell \Sigma \delta \rho g z+\Delta E
$$

where $\Delta E$ is the difference in surface free energy associated with the channel opening. We have

$$
\Delta E=\ell\left\{\sigma_{\mathrm{LW}} 2 w+\sigma_{\mathrm{LS}} 2 R\left[\frac{\pi}{2}-\left(\theta+\theta_{\mathrm{c}}\right)\right]-\sigma_{\mathrm{SW}} 2 w-\sigma_{\mathrm{GB}} e\right\} .
$$

This expression can be rewritten using (8), (9), and (10):

$$
\Delta E=2 \sigma_{\mathrm{LS}} R\left[\frac{\pi}{2}-\left(\theta+\theta_{\mathrm{c}}\right)+\sin \theta \cos \theta+\sin \theta_{\mathrm{c}} \cos \theta_{\mathrm{c}}-2 \cos \theta \cos \theta_{\mathrm{c}}\right] .
$$

Finally, (11), (13) and (15) lead to:

$$
\begin{aligned}
\frac{\Delta \Omega}{\ell \delta \rho g}= & {\left[\left(\cos \theta_{\mathrm{c}}-\sin \theta\right)\left(\cos \theta-\sin \theta_{\mathrm{c}}\right)+\cos \left(\theta+\theta_{\mathrm{c}}\right)+\theta+\theta_{\mathrm{c}}-\frac{\pi}{2}\right] } \\
& \times\left(z R^{2}-2 l_{\mathrm{c}}^{2} R\right) .
\end{aligned}
$$

$\Delta \Omega$ has a strictly negative minimum as a function of $R$, provided that $\theta+\theta_{\mathrm{c}}<\pi / 2$. It is reached for $R=l_{\mathrm{c}}^{2} / z$, confirming the previous result. In addition, this shows that the channel is stable under the above conditions, and that there is no energy barrier separating it from the state with no channel: it forms spontaneously.

We can now specify the region where the above relations are valid. The ratio between the vertical and horizontal curvatures is

$$
\eta=\frac{R(z) e^{\prime \prime}(z)}{\left[1+e^{\prime}(z)^{2}\right]^{3 / 2}}=\frac{2 C \zeta}{\left(1+C^{2} \zeta\right)^{3 / 2}}
$$

with $C=\cos \theta_{\mathrm{c}}-\sin \theta$ and $\zeta=\left(l_{\mathrm{c}} / z\right)^{4}$. To illustrate the results, we will use the following numbers: $\rho_{\mathrm{L}}=172.9 \mathrm{~kg} \mathrm{~m}^{-3}, \rho_{\mathrm{S}}=190.8 \mathrm{~kg} \mathrm{~m}^{-3}, \sigma_{\mathrm{LS}}=0.17 \mathrm{mN} \mathrm{m}^{-1}$, $\theta_{\mathrm{c}}=\pi / 4, \theta=\pi / 12$. Then $l_{\mathrm{c}}=986 \mu \mathrm{m}$, and $\eta$ becomes less than 0.1 for $z / l_{\mathrm{c}}>1.72$. Figure 16a shows $e$ and $w$ deduced from (9) and (10) at equilibrium (with $R=l_{\mathrm{c}}^{2} / z$ ), valid for $z>1.69 \mathrm{~mm}$. The functional form $w \propto 1 / z$ has been verified experimentally for several samples [12,27], with coefficients close to the one obtained with the above values.

We can also apply the present analysis to the study of a GB in contact with a wall at $P_{\mathrm{S}}$ above $P_{\text {eq }}$. In this case, the channel can remain in a liquid state up to a certain pressure which can be calculated. The equilibrium condition is deduced from the equality of the chemical potentials ((12), where we now neglect the gravity):

$$
\mu_{\mathrm{L}}\left(P_{\mathrm{L}}\right)=\mu_{\mathrm{L}}\left(P_{\mathrm{eq}}\right)+\frac{P_{\mathrm{L}}-P_{\mathrm{eq}}}{\rho_{\mathrm{L}}}=\mu_{\mathrm{S}}\left(P_{\mathrm{S}}\right)=\mu_{\mathrm{S}}\left(P_{\mathrm{eq}}\right)+\frac{P_{\mathrm{S}}-P_{\mathrm{eq}}}{\rho_{\mathrm{S}}}
$$


Fig. 16 Variation of the channel dimensions $e$ (solid line) and $w$ (dashed line) for the numbers given in the text, as a function of the depth at liquid-solid equilibrium (a), and as a function of pressure (b). $P_{\mathrm{S}}$ is the ambient solid pressure and $P_{\mathrm{m}}=P_{\mathrm{eq}}$ is the "melting" or liquid-solid equilibrium pressure
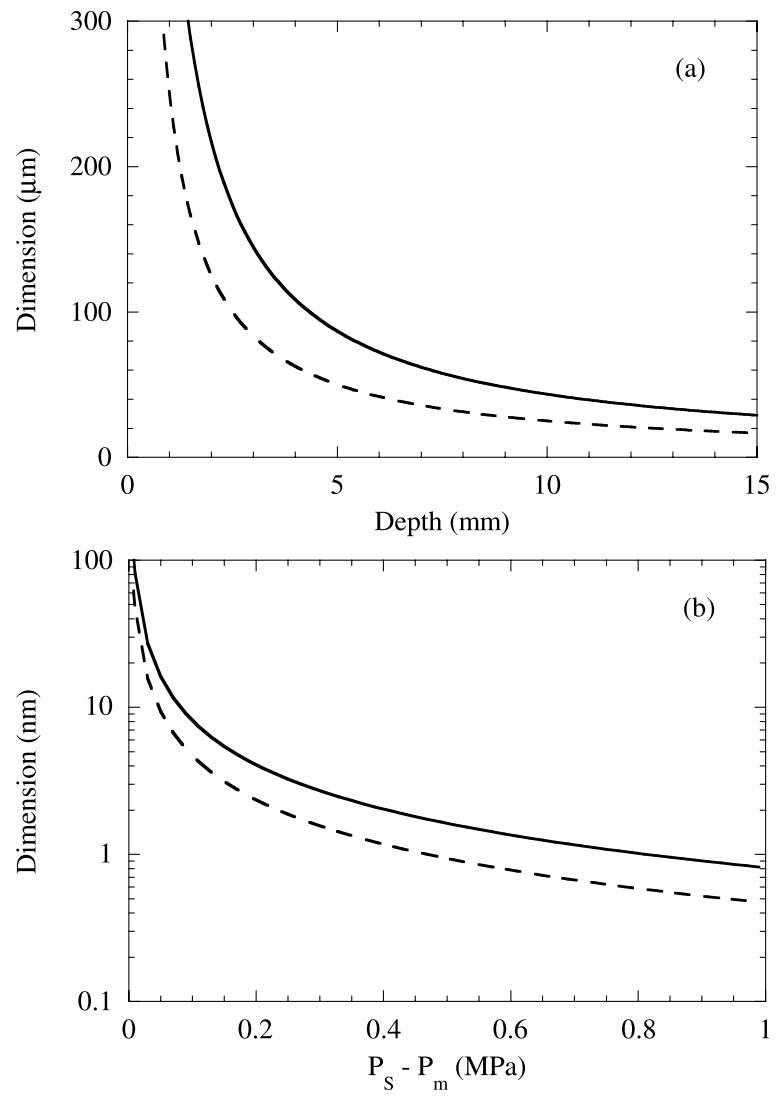

that is

$$
P_{\mathrm{L}}=\frac{\rho_{\mathrm{L}}}{\rho_{\mathrm{S}}} P_{\mathrm{S}}+\left(1-\frac{\rho_{\mathrm{L}}}{\rho_{\mathrm{S}}}\right) P_{\mathrm{eq}} .
$$

The radius of curvature of the interface follows from Laplace relation, $R=$ $\sigma_{\mathrm{LS}} /\left(P_{\mathrm{S}}-P_{\mathrm{L}}\right)$, and $e$ and $w$ are deduced from (9) and (10). Figure $16 \mathrm{~b}$ shows that the size of the liquid channel decreases rapidly when $P_{\mathrm{S}}$ increases. The typical pressure at which it closes is when $2 w$ reaches $1 \mathrm{~nm}$, the typical thickness of a GB. This occurs $0.94 \mathrm{MPa}$ above $P_{\text {eq }}$. Note that elasticity was neglected: by increasing the energy of the solid phase, local stresses could stabilize wider liquid channels.

\subsection{Liquid Channels where Three Grains Meet}

Let us now consider three crystal grains. They define three GBs that meet at a contact line. For the same reason as considered above, this contact line will be invaded by the liquid phase if $2 \theta$ is less than $60^{\circ}$ (Fig. 17). As explained above, $2 \theta$ is generically around $30^{\circ}$. We thus expect the cross section of the liquid channel to be a curved 
Fig. 17 A cross section of the liquid channel which should exist at the crossing of three GBs (dash-dotted lines), that is between three grains. The angle $2 \theta$ is taken as about $30^{\circ}$ in agreement with our measurements (see Sect. 4.2). The curvature radius $R$ is inversely proportional to the departure from the liquid-solid equilibrium pressure $P_{\mathrm{m}}$

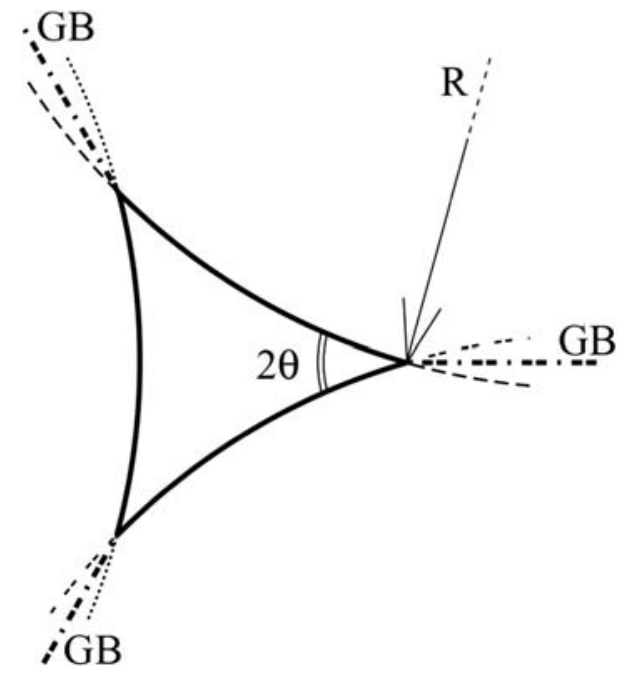

Fig. 18 The cross section area of liquid channels as a function of the departure from the melting pressure

$P_{\mathrm{m}}=2.53 \mathrm{MPa}$. These channels exist both where GBs meet walls (solid line) and where GBs meet each other as on Fig. 17 (dashed line)

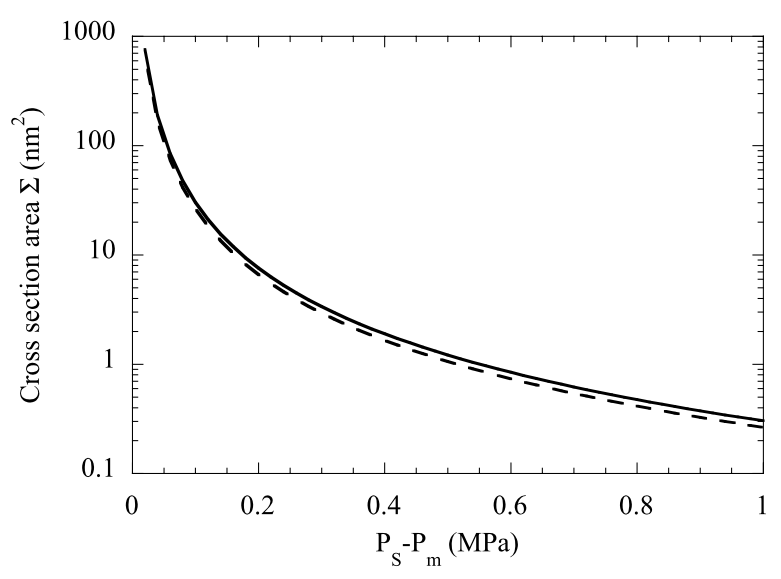

triangle whose area is

$$
\Sigma=R^{2}\left[2 \sqrt{3} \sin \phi \sin \left(\phi+\frac{\pi}{3}\right)-3 \phi\right],
$$

where

$$
R=\frac{\rho_{\mathrm{S}}}{\rho_{\mathrm{S}}-\rho_{\mathrm{L}}} \frac{\sigma_{\mathrm{LS}}}{P_{\mathrm{S}}-P_{\mathrm{eq}}}
$$

is the radius of curvature of the liquid-solid interface, and $\phi=\pi / 6-\theta \approx \pi / 12 \mathrm{rad}$. Figure 18 compares $\Sigma\left(P_{\mathrm{S}}-P_{\mathrm{eq}}\right)$ for a liquid channel on a wall and for a liquid channel between grains.

This effect had already been predicted by Miller and Chadwick [73] who expressed the departure from the melting curve in terms of temperature instead of pressure, but this is equivalent. A polycrystal near $P_{\text {eq }}$ should thus be invaded by 
Fig. 19 The time variation of the height $h(t)$ in the experiment by Sasaki et al. [10, 11], as predicted by (28). The height $h$ is measured from the bottom of the tube. The relaxation starts at $h=10 \mathrm{~mm}$ and stops at $h=5 \mathrm{~mm}$ where equilibrium is reached with the level outside the tube

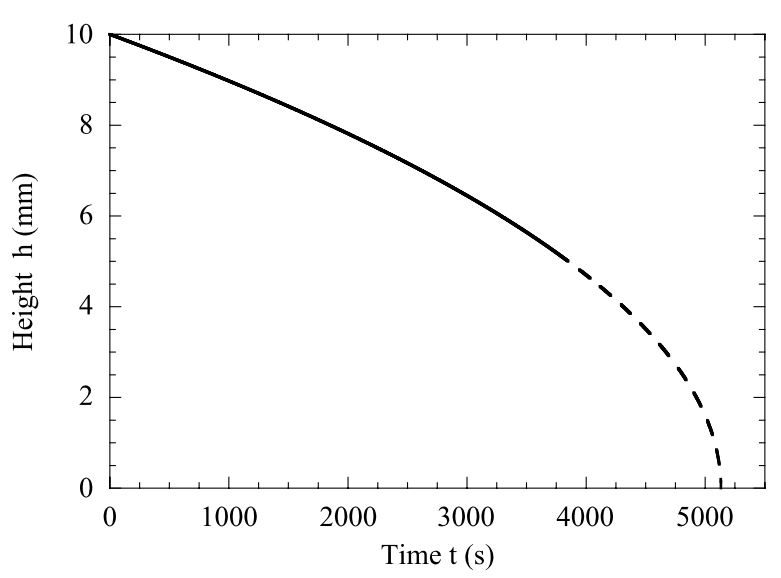

a three-dimensional network of liquid channels. Such a network has been observed in ice crystals [72]. Each node of this network will be a liquid droplet. If the pressure increases above $P_{\text {eq }}$, the channels and droplets will shrink and close around $1 \mathrm{MPa}$ above $P_{\text {eq }}$ when their size becomes of atomic dimension (see Fig. 19), unless they are stabilized by local stresses. When analyzing the superfluid properties of polycrystals, the existence of such liquid regions should be kept in mind.

\section{Discussion of Mass Flow in the Presence of GBs}

We now discuss the relevance of these results for the supersolid behavior observed in the mass flow experiment of Sasaki et al. [10, 11]. In the experimental setup, solid helium exists in equilibrium with the liquid inside and outside a tube closed at the top. The temperature being homogeneous, the pressure at the flat LS interfaces inside and outside the tube must be $P_{\text {eq }}$. If the two interfaces are at different heights, a flow should then be induced until they reach the same height. For good quality crystals, no movement of the LS interfaces was detected within experimental resolution: this was interpreted as the absence of supersolidity in perfect crystals at $50 \mathrm{mK}[10,11]$.

On the other hand, the levels of the two LS interfaces relaxed if a GB was present in the tube. Let us call $h$ the distance between the LS interface in the tube and the bottom of the tube. The relaxation of $h$ occurred at a nearly constant velocity $\mathrm{d} h / \mathrm{d} t=$ $-0.6 \mu \mathrm{m} \mathrm{s}^{-1}$ for crystal 1 . Note that the velocity depends on the crystal: crystal 2 started to relax with $\mathrm{d} h / \mathrm{d} t \simeq-6 \mu \mathrm{m} \mathrm{s}^{-1}$; as crystal 2 showed more cusps at the LS interface, this may be due to the existence of several paths available for flow. We will use $\mathrm{d} h / \mathrm{d} t=-0.6 \mu \mathrm{m} \mathrm{s}^{-1}$ in the following. Assuming that the relaxation is due to superfluid flow inside the GB, conservation of the mass flow leads to

$$
\Delta \rho A \frac{\mathrm{d} h}{\mathrm{~d} t}=-\rho_{\mathrm{GB}} \Sigma_{\mathrm{GB}} v_{\mathrm{c}}^{\mathrm{GB}}
$$

where $A=\pi D^{2} / 4$ where $D=10 \mathrm{~mm}$, and $\Sigma_{\mathrm{GB}}$ are the horizontal cross section area of the tube and GB, respectively, $\rho_{\mathrm{GB}}$ and $v_{\mathrm{c}}^{\mathrm{GB}}$ are the density and the critical 
velocity of the superfluid flow inside the GB. With $A=78.5 \mathrm{~mm}^{2}, \rho_{\mathrm{GB}}=\rho_{\mathrm{L}}$, and $\Sigma_{\mathrm{GB}}$ estimated to be $10 \times 0.3 \mathrm{~nm}=3 \mu \mathrm{m}^{2}$, we obtained $v_{\mathrm{c}}^{\mathrm{GB}} \simeq 1.6 \mathrm{~m} \mathrm{~s}^{-1}[10,11]$. This value is consistent with the one measured for flow in liquid helium films of atomic thickness adsorbed on a wall [74].

Now, according to the present results, if a GB is present, two liquid channels can also be present along the tube wall (depending on the values of $\theta$ and $\theta_{\mathrm{c}}$ ) and allow superfluid flow in the liquid. The cross section of one channel $\Sigma_{\mathrm{ch}}$ varies with the height $z$ above the bottom of the tube, and so does the flow velocity $v$, according to the relation for flow conservation: $v(z) \Sigma_{\mathrm{ch}}(z)=$ cte. If the critical velocity varies more slowly than $1 / \Sigma_{\text {ch }}$ (see below), then the flow reaches the critical velocity in the narrowest part of the channel (at the bottom of the tube) and has a velocity lower than critical everywhere else.

One may wonder how the shape of the channel will evolve during relaxation. Because of the high mobility of the LS interface in helium at low temperature, we expect the channel shape to adapt to insure the equality of the chemical potentials on both sides of the LS interface. Indeed, when the outer LS interface is lowered, it induces a chemical potential jump of order $\Delta \mu g h$. At low temperature, the interface velocity would be around $100 \Delta \mu$ [19], that is $\simeq 1 \mathrm{~m} \mathrm{~s}^{-1}$ for $h \simeq 10 \mathrm{~mm}$, which is much faster than the observed relaxation velocity. We see that the relaxation is limited by the critical velocity in the channel, not by the mobility of the liquid-solid interface.

Of course, the chemical potential cannot be homogeneous, because there would then be no flow at all. But we may assume that, because of the slow relaxation, near equilibrium conditions are achieved inside the tube. In addition, we will neglect elastic effects. At any height $z$, the pressure $P_{\mathrm{S}}(z)$ in the solid and the pressure $P_{\mathrm{L}}(z)$ in the liquid are related through (19).

We use the Bernoulli relation for the flow in the channel:

$$
P_{\mathrm{L}}(z)+\frac{1}{2} \rho_{\mathrm{L}} v(z)^{2}+\rho_{\mathrm{L}} g z=P_{\mathrm{eq}}+\rho_{\mathrm{L}} g h .
$$

The pressure difference across the LS interface at height $z$ is balanced by the surface tension. The radius of curvature $R(z)$ is thus given by the Laplace relation:

$$
\frac{\sigma_{\mathrm{LS}}}{R(z)}=P_{\mathrm{S}}(z)-P_{\mathrm{L}}(z)=\Delta \rho g h+\frac{1}{2} \rho_{\mathrm{L}} v(z)^{2} .
$$

Compared to the static situation, the flow reduces the liquid pressure (Bernoulli effect), and the LS interface becomes more curved. $\Sigma_{\mathrm{ch}}$ and $R$ are related through $\Sigma_{\mathrm{ch}}=K R^{2}$ with the constant $K$ defined by (11), so that the channel section becomes smaller. For $\theta_{\mathrm{c}}=\pi / 4$ and $\theta=\pi / 12, K=0.092$.

We now discuss the relative effect of the gravity and Bernoulli contributions. For $h=10 \mathrm{~mm}$, they become equal for a velocity $v_{1}=0.14 \mathrm{~m} \mathrm{~s}^{-1}$. Let us estimate the critical velocity we expect in the liquid channel. For a cylindrical channel of diameter $d$, we may use the Feynmann relation [75]:

$$
v_{\mathrm{c}}(d)=\frac{\kappa_{4}}{2 \pi d} \ln \left(\frac{d}{a_{0}}\right)
$$


where $\kappa_{4}=2 \pi \hbar / m_{4} \simeq 10^{-7} \mathrm{~m}^{2} \mathrm{~s}^{-1}$ is the quantum of circulation, and $a_{0} \simeq 0.1 \mathrm{~nm}$ is the radius of a vortex core. We assume that the critical velocity in a channel of section $\Sigma_{\mathrm{ch}}$ is the same as the one of a cylindrical channel with the same cross-section, that is with diameter $d=\sqrt{4 \Sigma_{\mathrm{ch}} / \pi}$. For the typical values of $\Sigma_{\mathrm{ch}}$, the logarithm in (25) can be taken as a constant around 13 , so that:

$$
v_{\mathrm{c}}\left(\Sigma_{\mathrm{ch}}\right)=\frac{\alpha}{\sqrt{\Sigma_{\mathrm{ch}}}}
$$

with $\alpha=1.8 \times 10^{-7} \mathrm{~m}^{2} \mathrm{~s}^{-1}$. Using the value $\Sigma_{\mathrm{ch}}=866 \mu \mathrm{m}^{2}$ for a channel at equilibrium at a depth $10 \mathrm{~mm}$, we expect a critical velocity of $6 \mathrm{~mm} \mathrm{~s}^{-1}$. This is much smaller than $v_{1}$ and the Bernoulli term in (24) may be neglected. The shape of the channel remains the same as in the static situation, with $R=l_{\mathrm{c}} / h$.

In that case, mass flows along the channels according to

$$
\Delta \rho A \frac{\mathrm{d} h}{\mathrm{~d} t}=-2 \rho_{\mathrm{L}} \Sigma_{\mathrm{ch}}(h) v_{\mathrm{c}}\left[\Sigma_{\mathrm{ch}}(h)\right] .
$$

The factor 2 stems from the existence of two liquid channels for $1 \mathrm{~GB}$. This leads to

$$
h(t)=h_{0}\left[1-\frac{t}{\tau}\left(\frac{l_{\mathrm{c}}}{h_{0}}\right)^{2}\right]^{1 / 2}
$$

where $h_{0}$ is the initial height of the LS interface inside the tube, and

$$
\tau=\frac{\Delta \rho}{\rho_{\mathrm{L}}} \frac{A}{4 \alpha \sqrt{K}}
$$

$\tau=37 \mathrm{~s}$ with the above numbers. In the experiment, the LS interface inside the tube is initially at $h_{0} \simeq 10 \mathrm{~mm}$ above the bottom of the tube, and at $h_{1} \simeq h_{0} / 2 \simeq 5 \mathrm{~mm}$ above the LS interface outside the tube. The driving force for flow will disappear when $h(t)=h_{1}$, at a time

$$
t_{1}=\frac{3}{4}\left(\frac{h_{0}}{l_{\mathrm{c}}}\right)^{2} \tau \simeq 3850 \mathrm{~s} .
$$

The initial slope $\mathrm{d} h / \mathrm{d} t$ would be $-1.3 \mu \mathrm{m} \mathrm{s}^{-1}$ (see Fig. 19). This is of the same order of magnitude as the $-0.6 \mu \mathrm{m} \mathrm{s}^{-1}$ observed for crystal 1 , so that the observed flow can also be explained by flow along the liquid channels. Note that, in this experiment, the flow stopped before equilibrium was reached, presumably because the path for mass flow was suddenly interrupted $[10,11]$.

Even if the quantitative values of $\mathrm{d} h / \mathrm{d} t$ for each scenario are comparable, one would expect a qualitatively different behavior, with a flow at a constant rate along a GB, and an accelerated one along the liquid channels. Crystal 2 exhibits the latter behavior, but it could also be due to the LS interface entering a region with more disorder where more structures for flow become available. Based on the above analysis, we conclude that the previous experiment is not able to decide between mass flow 
along the GB or along the liquid channel. We note however that flow was also observed at $1.13 \mathrm{~K}$. As the GB is predicted to become superfluid around $0.5 \mathrm{~K}[13,14]$, this supports the liquid channel scenario. It would be interesting to change the common width of the tube and the GB. If the width is reduced, $\Sigma_{\mathrm{GB}}$ would be reduced while keeping $\Sigma_{\text {ch }}$ constant: if the flow takes place along the GB, the relaxation velocity should be unchanged, whereas if it occurs along the liquid channel, it should be increased.

As for the recent experiment by Ray and Hallock [15], we do not think that it provides a strong evidence for superflow of mass along GBs either [16]. The solid samples in which they have observed mass flow are at pressures $P$ in the range 2.60 to $2.68 \mathrm{MPa}$ which is only 0.07 to $0.15 \mathrm{MPa}$ above $P_{\text {eq }}=2.53 \mathrm{MPa}$. Our study shows that liquid channels should exist at each crossing of GBs with a width inversely proportional to $\left(P-P_{\mathrm{eq}}\right)$. In their sample $\mathrm{A}$ at $2.68 \mathrm{MPa}$, the cross section area of the liquid channels should be $12 \mathrm{~nm}^{2}$ (see Fig. 18). In reality, a density of about 10 channels per $\mathrm{mm}^{2}$ would be sufficient to explain their flow rate, corresponding to a polycrystalline sample with typical grain sizes of $0.35 \mathrm{~mm}$. This looks possible to us, so that mass could flow along liquid channels in their samples, and a robust evidence of superfluidity along GBs remains to be found.

We would like to mention another interesting possibility. The above analysis was based on the Feynmann formula for the critical velocity, (25). However, Varoquaux et al. [75], studying flow through small apertures, have obtained much higher values, above $v_{1}$. If this holds in our experimental situation, the Bernoulli term in (24) cannot be neglected, and it tends to reduce $\Sigma_{\text {ch }}$ and the corresponding flow. To give an idea, let us assume a constant critical velocity of $v_{2}=2 \mathrm{~m} \mathrm{~s}^{-1}$ for all values of $\Sigma_{\text {ch }}$ [75]. In that case, the gravity term in (24) can be neglected, and one finds

$$
\Sigma_{\mathrm{ch}}=4 K\left(\frac{\sigma_{\mathrm{LS}}}{\rho_{\mathrm{L}} v_{2}^{2}}\right)^{2} \simeq 0.02 \mu \mathrm{m}^{2} .
$$

According to (27), the relaxation should then occur at a rate $\mathrm{d} h / \mathrm{d} t \simeq-1.9 \mathrm{~nm} \mathrm{~s}^{-1}$, much smaller than experimentally observed. We conclude that either the critical velocity is really small, as expected from the Feynmann relation, or that the observed flow occurs along the GBs.

\section{Conclusion}

We have studied solid helium samples grown at constant volume and showed that they are polycrystals with a small grain size in the micrometer range. We have also studied grain boundaries and showed that their existence leads to the existence of liquid channels near walls and also inside polycrystals up to pressures about $1 \mathrm{MPa}$ above the liquid-solid equilibrium pressure $P_{\mathrm{eq}}=2.5 \mathrm{MPa}$. In the light of these observations we have reconsidered experiments on mass flow through solid helium. It appears that grain boundaries have to be considered in many experiments addressing the possible existence of supersolidity. However, there is no evidence yet for their superfluidity. We have also shown that grain boundaries have a microscopic thickness so that their 
inside is not bulk liquid. Pollet et al. $[13,14]$ have predicted that grain boundaries should become superfluid below a critical temperature of order a fraction of a Kelvin, depending on the orientation of the grains. It is a very interesting challenge to show that this superfluid transition exists in GBs which are new and original 2D-quantum systems. An illustration of their originality is as follows: contrary to a liquid where all the mass is superfluid in the zero temperature limit, some of the mass should remain localized and some of the mass should become superfluid in a disordered solid such as a grain boundary. This is what appears on Fig. 3 of the article by Pollet et al. [13, 14].

Acknowledgements We are grateful to P. Courtois (ILL Grenoble, France) who gave us graphite samples, to L. Battarra, N. Jamal and X. Rojas for help in the experiments and to H.J. Maris, P. Nozières, Yu. Mukharsky, E. Rolley and C. Caroli for fruitful discussions.

This work is supported by ANR grant 05-BLAN-0084-01 and ANR grant BLAN07-1-215296.

\section{References}

1. E. Kim, M.H.W. Chan, Nature (Lond.) 427, 225 (2004)

2. E. Kim, M.H.W. Chan, Science 305, 1941 (2004)

3. E. Kim, M.H.W. Chan, Phys. Rev. Lett. 97, 115302 (2006)

4. S. Balibar, F. Caupin, J. Phys.: Condens. Matter 20, 173201 (2008)

5. A.S.C. Rittner, J.D. Reppy, Phys. Rev. Lett. 97, 165301 (2006)

6. A. Penzev, Y. Yasuta, M. Kubota, J. Low Temp. Phys. 148, 677 (2007)

7. M. Kondo, S. Takada, Y. Shibayama, K. Shirahama, J. Low Temp. Phys. 148, 695 (2007)

8. Y. Aoki, J.C. Graves, H. Kojima, Phys. Rev. Lett. 99, 015301 (2007)

9. A.S.C. Rittner, J.D. Reppy, Phys. Rev. Lett. 98, 175302 (2007)

10. S. Sasaki, R. Ishiguro, F. Caupin, H.J. Maris, S. Balibar, Science 313, 1098 (2006)

11. S. Sasaki, R. Ishiguro, F. Caupin, H.J. Maris, S. Balibar, J. Low Temp. Phys. 148, 665 (2007)

12. S. Sasaki, F. Caupin, S. Balibar, Phys. Rev. Lett. 99, 205302 (2007)

13. L. Pollet, M. Boninsegni, A.B. Kuklov, N.V. Prokof'ev, B.V. Svistunov, M. Troyer, Phys. Rev. Lett. 98, 135301 (2007)

14. L. Pollet, M. Boninsegni, A.B. Kuklov, N.V. Prokof'ev, B.V. Svistunov, M. Troyer, Phys. Rev. Lett. 101, 097202 (2008)

15. M.W. Ray, R.B. Hallock, Phys. Rev. Lett. 100, 235301 (2008)

16. S. Balibar, F. Caupin, Phys. Rev, Lett. (July 2008), submitted

17. A.C. Clark, J.T. West, M.H.W. Chan, Phys. Rev. Lett. 99, 135302 (2007)

18. M.H.W. Chan, Private communication, Workshop "Supersolidity", Trieste, August 2008

19. S. Balibar, H. Alles, A. Ya, Parshin, Rev. Mod. Phys. 77, 317 (2005)

20. M. Boninsegni, A.B. Kuklov, L. Pollet, N. Prokof'ev, B. Svistunov, M. Troyer, Phys. Rev. Lett. 97, 080401 (2006)

21. M. Boninsegni, A.B. Kuklov, L. Pollet, N.V. Prokof'ev, B.V. Svistunov, M. Troyer, Phys. Rev. Lett. 99, 035301 (2007)

22. J. Toner, Phys. Rev. Lett. 100, 035302 (2008)

23. G. Biroli, J.P. Bouchaud, arXiv:cond-mat/0710.3087v2 (2007)

24. J. Day, J. Beamish, Nature 450, 853 (2007)

25. E. Kim, J.S. Xia, J.T. West, X. Lin, M.H.W. Chan, Bull. Am. Phys. Soc. 52, 610 (2007)

26. E. Kim, J.S. Xia, J.T. West, X. Lin, A.C. Clark, M.H.W. Chan, Phys. Rev. Lett. 100, 065301 (2008)

27. F. Caupin, S. Sasaki, S. Balibar, J. Low Temp. Phys. 150, 267 (2008)

28. J.K. Hoffer, W.R. Gardner, C.G. Waterfield, N.E. Phillips, J. Low Temp. Phys. 23, 63 (1976)

29. C.A. Swenson, Phys. Rev. 79, 626 (1950)

30. C.A. Swenson, Phys. Rev. 89, 538 (1953)

31. J.H. Vignos, H.A. Fairbank, Phys. Rev. 147, 185 (1966)

32. D.O. Edwards, R.C. Pandorf, Phys. Rev. 140A, 816 (1965)

33. J. Day, J. Beamish, Phys. Rev. Lett. 96, 105304 (2006)

34. H.J. Maris, F. Caupin, J. Low Temp. Phys. 131, 145 (2003) 
35. V.M. Giordano, F. Datchi, Phys. Rev. Lett. 99, 165701 (2007)

36. E.R. Grilly, J. Low Temp. Phys. 11, 33 (1973)

37. R.J. Asaro, W.A. Tiller, Metall. Trans. A 3, 1789 (1972)

38. M.A. Grinfeld, Dokl. Akad. Nauk SSSR 290, 1358 (1986). [Sov. Phys. Dokl. 31, 831 (1986)]

39. P. Nozières, Shape and growth of crytals, in Solids Far from Equilibrium, ed. by C. Gordrèche (Cambridge University Press, Cambridge, 1992)

40. S. Balibar, D.O. Edwards, W.F. Saam, J. Low Temp. Phys. 82, 119 (1991)

41. J.N. Aqua, T. Frisch, A. Verga, Phys. Rev. B 76, 165319 (2007)

42. J. Bodensohn, K. Nicolai, P. Leiderer, Z. Phys. B: Condens. Matter 64, 55 (1986)

43. M. Thiel, A. Willibald, P. Evers, A. Levchenko, P. Leiderer, S. Balibar, Europhys. Lett. 20, 707 (1992)

44. R. Torii, S. Balibar, J. Low Temp. Phys. 89, 391 (1992)

45. A. Pocheau, M. Georgelin, Phys. Rev. E 73, 011604 (2006) and references therein

46. M.H.W. Chan, Science 319, 1207 (2008)

47. F.J. Webb, K.R. Wilkinson, J. Wilks, Proc. R. Soc. A 214, 546 (1952)

48. G.A. Armstrong, A.A. Helmy, A.S. Greenberg, Phys. Rev. B 20, 1061 (1979)

49. N. Mulders, Oral communication, Workshop "Supersolidity", Trieste, August 2008

50. Yu. Mukharsky, Oral communication, Workshop "Supersolidity", Trieste, August 2008

51. A.F. Schuch, R.F. Mills, Phys. Rev. Lett. 8, 369 (1962)

52. M. Maekawa, Y. Okumura, Y. Okuda, Phys. Rev. B 65, 144525 (2002)

53. N.C. Ford Jr., R. Hallock, K.H. Langley, J. Low Temp. Phys. 148, 653 (2007)

54. H.J. Junes, H. Alles, M.S. Manninen, A.Ya. Parshin, I.A. Todoshchenko, J. Low Temp. Phys. (2008, to appear)

55. L.D. Landau, I.M. Lifshitz, Fluid mechanics, in Theoretical Physics, vol. 6 (Butterworth, Stoneham, 1987), Chap. VII

56. P.-G. de Gennes, F. Brochard-Wyart, D. Quéré, Capillarity and Wetting Phenomena: Drops, Bubbles, Pearls, Waves (Springer, Berlin, 2003), Chap. 2, Sect. 3.2

57. G.F. Bolling, W.A. Tiller, J. Appl. Phys. 31, 1345 (1960)

58. U. Böyük, K. Keşlioğlu, N. Maraşh, J. Phys. Condens. Matter 19, 116202 (2007) and references therein

59. R. Kikuchi, J.W. Cahn, Phys. Rev. B 21, 1893 (1980)

60. T.E. Hsieh, R.W. Balluffi, Acta Metall. 37, 1637 (1989)

61. A.M. Alsayed, M.F. Islam, J. Zhang, P.J. Collings, A.G. Yodh, Science 309, 1207 (2005)

62. R. Lipowsky, Phys. Rev. Lett. 57, 2876 (1986)

63. M. Schick, W.-H. Shih, Phys. Rev. B 35, 5030 (1987)

64. S. Dietrich, Wetting phenomena, in Phase Transitions and Critical Phenomena, ed. by C. Domb, J.L. Lebowitz, vol. 12 (Academic Press, San Diego, 1988)

65. F. Caupin, S. Sasaki, S. Balibar, Proc. of the Liquids Conference (Lund, 2008), J. Phys. Condens. Matter, Special issue (Nov. 2008), to appear

66. J.P. Franck, K.E. Kornelsen, J.R. Manuel, Phys. Rev. Lett. 50, 1463 (1983)

67. J.P. Franck, J. Gleeson, K.E. Kornelsen, J.R. Manuel, K.A. McGreer, J. Low Temp. Phys. 58, 153 (1985)

68. J.G. Dash, H.Y. Fu, J.S. Wetlaufer, Rep. Prog. Phys. 58, 115 (1995)

69. J.G. Dash, J.S. Wetlaufer, Phys. Rev. Lett. 94, 235301 (2005)

70. E. Rolley, C. Guthmann, Phys. Rev. Lett. 98, 166105 (2007)

71. S. Balibar, B. Castaing, C. Laroche, J. Phys. Lett. 41, 283 (1980)

72. A.W. Rempel, E.D. Waddington, J.S. Wetlaufer, M.G. Worster, Nature 411, 568 (2001)

73. W.A. Miller, G.A. Chadwick, Acta Metall. 15, 607 (1967)

74. K. Telschow, I. Rudnick, T.G. Wang, Phys. Rev. Lett. 32, 1292 (1974)

75. E. Varoquaux, C. R. Phys. 7, 1101 (2006) 